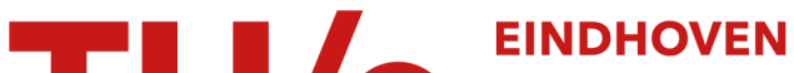 \\ UNIVERSITY OF \\ TECHNOLOGY
}

\section{Scalar transport in inline mixers with spatially periodic flows}

\section{Citation for published version (APA):}

Baskan, Ö., Rajaei, H., Speetjens, M. F. M., \& Clercx, H. J. H. (2017). Scalar transport in inline mixers with spatially periodic flows. Physics of Fluids, 29(1), [013601]. https://doi.org/10.1063/1.4973327

DOI:

$10.1063 / 1.4973327$

Document status and date:

Published: 01/01/2017

\section{Document Version:}

Publisher's PDF, also known as Version of Record (includes final page, issue and volume numbers)

\section{Please check the document version of this publication:}

- A submitted manuscript is the version of the article upon submission and before peer-review. There can be important differences between the submitted version and the official published version of record. People interested in the research are advised to contact the author for the final version of the publication, or visit the $\mathrm{DOI}$ to the publisher's website.

- The final author version and the galley proof are versions of the publication after peer review.

- The final published version features the final layout of the paper including the volume, issue and page numbers.

Link to publication

\section{General rights}

Copyright and moral rights for the publications made accessible in the public portal are retained by the authors and/or other copyright owners and it is a condition of accessing publications that users recognise and abide by the legal requirements associated with these rights.

- Users may download and print one copy of any publication from the public portal for the purpose of private study or research.

- You may not further distribute the material or use it for any profit-making activity or commercial gain

- You may freely distribute the URL identifying the publication in the public portal.

If the publication is distributed under the terms of Article 25fa of the Dutch Copyright Act, indicated by the "Taverne" license above, please follow below link for the End User Agreement:

www.tue.nl/taverne

Take down policy

If you believe that this document breaches copyright please contact us at:

openaccess@tue.nl

providing details and we will investigate your claim. 


\section{Scalar transport in inline mixers with spatially periodic flows}

Ozge Baskan, Hadi Rajaei, Michel F. M. Speetjens, and Herman J. H. Clercx

Citation: Phys. Fluids 29, 013601 (2017); doi: 10.1063/1.4973327

View online: http://dx.doi.org/10.1063/1.4973327

View Table of Contents: http://aip.scitation.org/toc/phf/29/1

Published by the American Institute of Physics

\section{Articles you may be interested in}

Vortex identification from local properties of the vorticity field

Phys. Fluids 29, 015101 (2017); 10.1063/1.4973243

The effect of surface charge convection and shape deformation on the settling velocity of drops in nonuniform electric field

Phys. Fluids 29, 012101 (2017); 10.1063/1.4973399

Effects of grid geometry on non-equilibrium dissipation in grid turbulence

Phys. Fluids 29, 015102 (2017); 10.1063/1.4973416

Aerodynamics of two-dimensional flapping wings in tandem configuration

Phys. Fluids 28, 121901 (2016); 10.1063/1.4971859 


\title{
Scalar transport in inline mixers with spatially periodic flows
}

\author{
Ozge Baskan, ${ }^{1}$ Hadi Rajaei, ${ }^{1}$ Michel F. M. Speetjens, ${ }^{2}$ and Herman J. H. Clercx ${ }^{1}$ \\ ${ }^{1}$ Fluid Dynamics Laboratory, Department of Applied Physics, Eindhoven University of Technology, \\ P.O. Box 513, 5600 MB Eindhoven, The Netherlands \\ ${ }^{2}$ Energy Technology Laboratory, Department of Mechanical Engineering, Eindhoven University of Technology, \\ P.O. Box 513, 5600 MB Eindhoven, The Netherlands
}

(Received 8 September 2016; accepted 13 December 2016; published online 6 January 2017)

\begin{abstract}
Spatially persisting patterns form during the downstream evolution of passive scalars in threedimensional (3D) spatially periodic flows due to the coupled effect of stretching and folding mechanisms of the flow field. This has been investigated in many computational and theoretical studies of 2D time-periodic and 3D spatially periodic flow fields. However, experimental studies, to date, have mainly focused on flow visualization with streaks of dye rather than fully $3 \mathrm{D}$ scalar field measurements. Our study employs 3D particle tracking velocimetry and 3D laser-induced fluorescence to analyze the evolution of 3D flow and scalar fields and the correlation between the coherent flow/scalar field structures in a representative inline mixer, the Quatro static mixer. For this purpose an experimental setup that consists of an optically accessible test section with transparent internal elements accommodating a pressure-driven pipe flow has been built. The flow and scalar fields clearly underline the complementarity of the experimental results with numerical simulations and provide validation of the periodicity assumption needed in numerical studies. The experimental procedure employed in this investigation, which allows studying the scalar transport in the advective limit, demonstrates the suitability of the present method for exploratory mixing studies of a variety of mixing devices, beyond the Quatro static mixer. Published by AIP Publishing. [http://dx.doi.org/10.1063/1.4973327]
\end{abstract}

\section{INTRODUCTION}

Advective-diffusive scalar transport in periodic laminar flows is of practical relevance to various industrial thermofluid processes that employ the inline mixing principle. Two important practical categories are blending of highly viscous fluids (e.g., polymers and food) $)^{1,2}$ and micro-fluidics (e.g., labon-a-chip technologies and bio-medical devices). ${ }^{3,4}$ Today's technology in inline mixing systems is based on empirical knowledge, yet further advancement requires deeper insight into transport mechanisms. In the past few decades, the fundamentals and applications of mass/scalar transport in spatially periodic laminar flows have been studied in numerous theoretical and numerical investigations. ${ }^{5-12}$ Nevertheless, a complete understanding is not achieved yet. Particularly, experimental studies are limited, i.e., restricted to highly idealized flow geometries or allowing only partial access to the flow domain with (optical) diagnostic tools. ${ }^{5,13-16}$ The work presented here aims to bridge this gap via laboratory experiments in a real mixer geometry.

This study adopts the Quatro mixer (Primix BV, Mijdrecht, The Netherlands) shown in Fig. 1 as a representative inline mixer for an in-depth analysis of advective-diffusive transport of scalars in industrial processes and the analysis expands on the work by Jilisen et al. ${ }^{17}$ The Quatro mixer geometry is composed of a cylinder that holds mixing elements consisting of chevron-shaped central plates with perpendicular elliptical parts extending to the inner wall of the cylinder. Each mixing element is a reflected and rotated (by $90^{\circ}$ ) version of the preceding one. Sequential placement of the reoriented elements inside the cylinder yields a downstream periodic repetition of element pairs, where two elements form one period. This results in a spatially periodic flow field inside the mixer. ${ }^{9}$

The main objective of the current study is to investigate scalar transport under the action of a spatially periodic laminar flow field. The study employs a combined experimentalnumerical analysis for the investigation of only-advective and of advective-diffusive scalar transport in the Quatro mixer. For the experimental analysis, a setup, which mimics realistic (industrial) flow conditions, is developed. The flow and concentration fields are measured by three-dimensional particle tracking velocimetry (3D PTV) and three-dimensional laserinduced fluorescence (3D LIF) techniques, respectively. For the numerical analysis, a finite-element method is employed. Studying scalar transport numerically in the advective limit, where diffusion is either absent or very weak compared to advective transport, is very challenging due to artificial diffusion introduced by the numerical discretization. In this respect, the experimental analysis is essential as it enables the investigation of the scalar transport in the advective limit.

\section{MODELLING FLOW AND SCALAR TRANSPORT}

The configuration is a steady, laminar, incompressible flow field and its related steady advective-diffusive scalar field inside the 3D mixer geometry, an element of which has a diameter $D$ and a length $L$ (see Fig. 1) with, in our case, $D=L$. The governing non-dimensional mass and momentum 

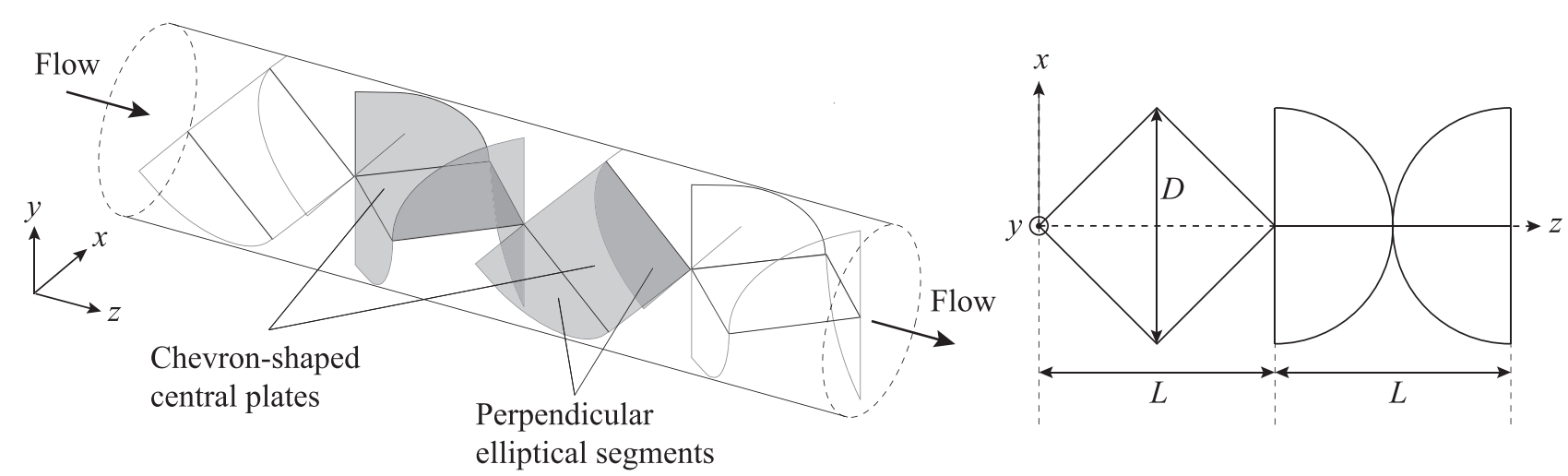

FIG. 1. A schematic of the Quatro mixer. The left panel shows the geometry of the Quatro mixer with the chevron-shaped central plates, perpendicular elliptical parts extending to the cylinder wall and the axial throughflow. The right panel shows the periodic segment of the mixer, the definition of the cylindrical coordinate system, the cylinder diameter, and the element width $D$ and the element length $L$. In the current study $D=L$.

equations are

$$
\boldsymbol{\nabla} \cdot \boldsymbol{u}=0, \operatorname{Re} \boldsymbol{u} \cdot \boldsymbol{\nabla} \boldsymbol{u}=-\boldsymbol{\nabla} P+\boldsymbol{\nabla}^{2} \boldsymbol{u},
$$

where $\boldsymbol{u}$ is the fluid velocity and $P$ the pressure. The scalar field $C$ is made dimensionless according to

$$
C=\frac{\mathrm{C}-\min \mathrm{C}_{0}(\boldsymbol{x})}{\max \mathrm{C}_{0}(\boldsymbol{x})-\min \mathrm{C}_{0}(\boldsymbol{x})}
$$

with $C$ being the dimensionful scalar, $\min \mathrm{C}_{0}(\boldsymbol{x})$ and $\max \mathrm{C}_{0}(\boldsymbol{x})$ the minimum and maximum values of the initial scalar field $\mathrm{C}_{0}(\boldsymbol{x})$, respectively. The non-dimensional steady advectiondiffusion equation reads

$$
\boldsymbol{u} \cdot \boldsymbol{\nabla} C=\frac{1}{P e} \nabla^{2} C
$$

These equations are also supplemented with no-slip and zeroflux conditions at the walls. Poiseuille flow is prescribed at the inlet, whereas a constant pressure boundary condition is set at the outlet. Moreover, a heterogeneous concentration is prescribed at the inlet $z=0$,

$$
\left.C\right|_{z=0}=\left\{\begin{array}{ll}
1, & \text { if } y \geq 0 \\
0, & \text { if } y<0
\end{array} .\right.
$$

The 3D coordinate system is defined in such a way that the $(x, y)$-plane corresponds to the transverse direction, whereas the $z$-direction corresponds to the axial (and downstream) direction, see Fig. 1. In Eqs. (1) and (3), Re and $P e$ are the Reynolds and Péclet number, respectively, defined as

$$
R e=\frac{U D}{v}, \quad P e=\frac{U D}{\alpha},
$$

where $U$ and $D$ are the characteristic velocity of the flow field (i.e., mean velocity $U_{\text {mean }}$ ) and the diameter of the domain, respectively, $v$ is the kinematic viscosity, and $\alpha$ the material diffusivity. The flow is laminar (low Re regime) and the scalar transport is dominated by advection (high $P e$ case) in the current investigation.

The scalar field analysis focuses on purely advective transport (where $P e$ is extremely high). The experimental methods used in the current study are perfectly capable of analyzing such advective transport phenomena, also in the Quatro mixer which is representative of more generic cases. However, from a practical point of view, the numerical model can accurately be solved only up to about $P e=1000$ due to the restriction imposed by the numerical resolution.

\section{METHODOLOGY}

\section{A. Numerical simulations}

The finite element package Comsol Multiphysics $4.3 \mathrm{~b}$ is used to solve 3D mass and momentum equations (Eq. (1)) and the advection-diffusion equation (Eq. (3)). A mixer model with 10 periods (20 mixing elements) is built in Comsol, and an unstructured tetrahedral grid is constructed with approximately $30 \times 10^{6}$ elements. The numerical convergence and accuracy are verified by standard grid refinement tests. Integrations are performed with a second-order backward-difference scheme. A relative tolerance of $10^{-3}$ is prescribed for the velocity components of the steady flow field and the steady scalar field in the solver.

\section{B. Experimental facilities}

Two separate laboratory setups have been constructed for experimental analyses of the Quatro mixer shown in Fig. 1. The first setup is for measurement of flow, streamlines, and Lagrangian transport by way of 3D PTV, and the second setup is for visualization and measurement of scalar transport by way of 3D LIF. The basic structure and the working principle of both facilities are the same. The main difference is that they are equipped with different data acquisition systems.

The 3D PTV facility, a detailed description of which is given by Jilisen et al., ${ }^{17}$ is shown in Fig. 2 (left panel). In Ref. 17 water was used as the working fluid (with a flow rate $\dot{Q}=80 \mathrm{l} / \mathrm{h}$ and a mean axial velocity $U_{\text {mean }}=9 \mathrm{~mm} / \mathrm{s}$ ), which results in a Reynolds number $R e=U_{\text {mean }} D / v=505$ (kinematic viscosity $\left.v_{\text {water }}=10^{-6} \mathrm{~m}^{2} / \mathrm{s}\right)$. The current investigation employs silicone oil (with a density $\rho=970 \mathrm{~kg} / \mathrm{m}^{3}$ and a kinematic viscosity $\left.v=10^{-4} \mathrm{~m}^{2} / \mathrm{s}\right)$, lowering the Reynolds number to $3.5\left(\dot{Q}=56 \mathrm{l} / \mathrm{h}\right.$ and $\left.U_{\text {mean }}=6.3 \mathrm{~mm} / \mathrm{s}\right)$. Note that silicone oils having viscosities lower than $v=10^{-3} \mathrm{~m}^{2} / \mathrm{s}$ show Newtonian behavior. ${ }^{18}$

The second Quatro mixer facility, shown in Fig. 2 (right), is designed for 3D LIF measurements. It is an open-loop system that uses both plain and fluorescent silicone oils (with a density $\rho=970 \mathrm{~kg} / \mathrm{m}^{3}$ and a kinematic viscosity $v=10^{-4} \mathrm{~m}^{2} / \mathrm{s}$ ) as the working fluid. It has two top reservoirs, which hold plain oil and fluorescent oil, and a bottom reservoir which accumulates the mixed oil. The top reservoirs 

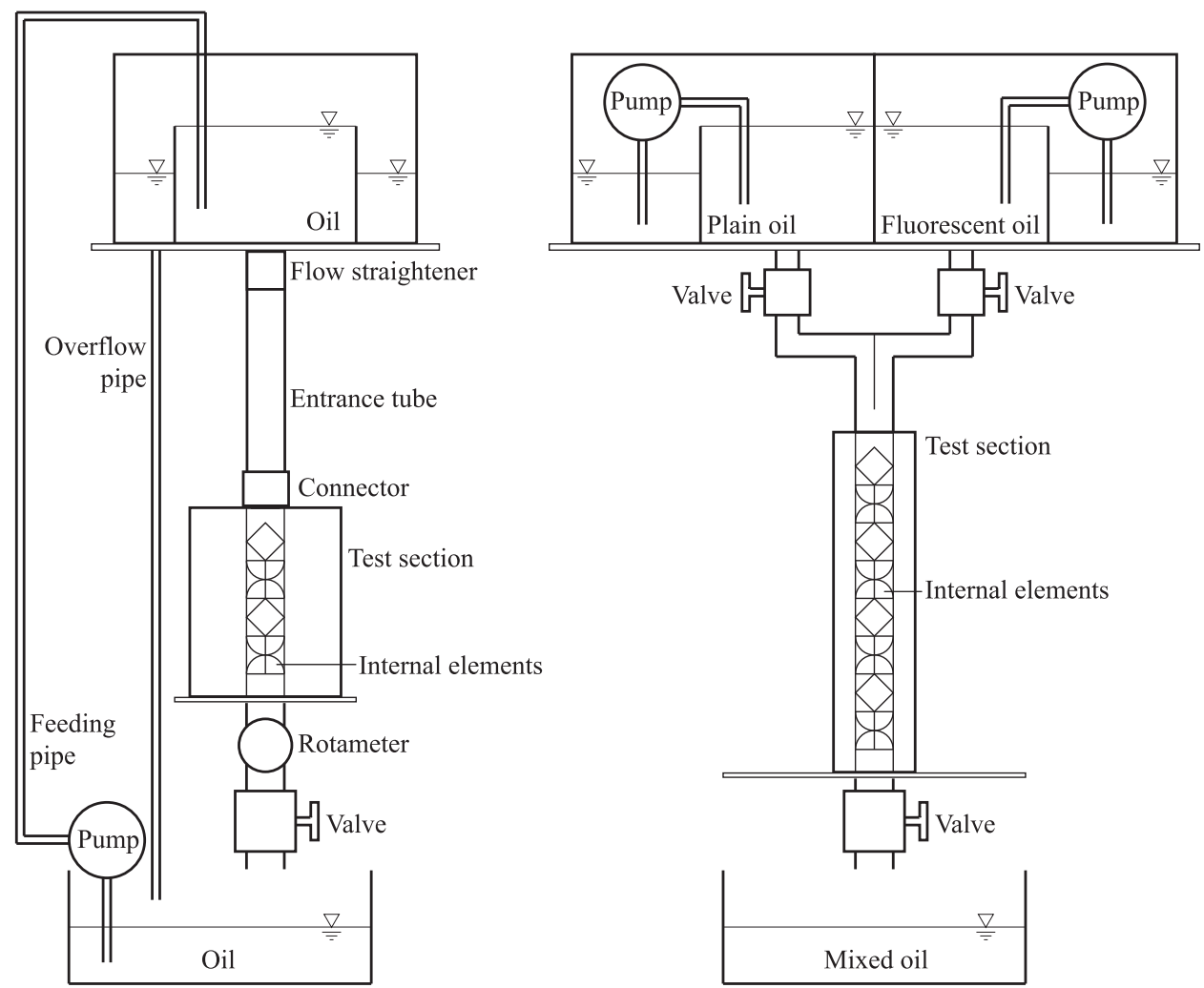

FIG. 2. A schematic sketch of the Quatro mixer facilities: the left and the right figures show the setups used for 3D PTV and 3D LIF measurements, respectively. Both facilities accommodate a transparent test section with a pressure-driven flow inside reservoirs and connecting tubes.

are divided into two parts to keep the flow rate constant inside the facility and the test section is made of the same materials as in Ref. 17 (the viewing box and the tube with an inner diameter $D=56 \mathrm{~mm}$ and a thickness $a=2 \mathrm{~mm}$ are glass and the mixing elements are Perspex with a thickness of $1.5 \mathrm{~mm}$ ). However, it is longer $(1 \mathrm{~m})$ than the one in the 3D PTV facility so as to accommodate more mixing elements and narrower (the cross-sectional area of the viewing box is a square with a side length of $100 \mathrm{~mm}$ ). Having a narrower viewing box does not affect the optical quality but significantly decreases the amount of liquid it holds. The mean velocity in the concentration field experiments is $U_{\text {mean }}=0.001 \mathrm{~m} / \mathrm{s}$, resulting in a Reynolds number $R e=0.5$, which is different compared to the Reynolds number in the flow field measurements (due to the restrictions imposed by practical issues).

The concentration field experiments are conducted at extremely high $P e$ (assuming the material diffusivity $\alpha$ is very weak). However, from a practical point of view, the numerical simulations of such mixing experiments can only be conducted accurately for Péclet numbers up to about $P e=1000$, above which the influence of numerical diffusion becomes substantial for the current numerical configuration (for Péclet numbers up to about $P e=1000$, the condition $P e_{\text {cell }}<2$ is satisfied in the current numerical configuration). ${ }^{19}$ Increasing mesh resolution, on the other hand, enables us to simulate the cases with higher Péclet numbers accurately, yet it is limited by the computational resources. Experiments and simulations are thus to a certain extent complementary as numerical simulations provide full detail of the mixing process, but for $P e \lesssim 1000$, while experiments enable us to investigate mixing up to very high Péclet numbers at the expense of global access to tracer distributions. Therefore, any comparative analysis between the experiments and the numerical simulations should focus on the features that can be accessed in both cases (e.g., largerscale structures and general correlation with the flow field) rather than one-to-one comparison between experiments and simulations.

\section{Measurement techniques}

\section{1. $3 D$ particle tracking velocimetry}

For 3D PTV experiments, the Quatro mixer facility is equipped with the same setup as in Ref. 17. Two arrays of light-emitting diodes (LEDs, LUXEON Rebel Color, Philips, The Netherlands) with an intensity peak at $530 \mathrm{~nm}$ wavelength are used for the illumination of the flow with fluorescent seeding particles [polymethylmethacrylate (PMMA) hollow sphere particles doped with Rhodamine-B; $d_{\mathrm{p}}=50 \mu \mathrm{m}$, $\rho_{\mathrm{p}}=1190 \mathrm{~kg} / \mathrm{m}^{3}$; Microparticles GmbH, Germany]. The particles have an absorption and an emission peak at $\lambda_{\mathrm{ab}}$ $=560 \mathrm{~nm}$ (green) and $\lambda_{\mathrm{em}}=585 \mathrm{~nm}$ (red), respectively. This enables the elimination of the reflections due to fluid-wall interfaces and small air bubbles, which deteriorates the image quality otherwise, by two-step optical filtering: cyan dichroic filters are mounted on the collimator lenses of the LEDs to exclude the LED contributions above $\lambda=570 \mathrm{~nm}$ that may interfere with the fluorescence and $590 \mathrm{~nm}$ high-pass filters (type OG-590, Schott Glass, Germany) are mounted on camera lenses to remove the reflections (around the LED peak) from the test section. The slight mismatch between the emission wavelength of the particles and the cut-off wavelength of the high-pass filter does not cause significant reduction in light intensity due to broad emission wavelength of the particles. 
The typical response time of the particles to changes in velocity field is estimated as $T_{\mathrm{p}}=d_{\mathrm{p}}^{2} \rho_{\mathrm{p}} /(18 \rho v)=1.7 \mu \mathrm{s}$, which is negligible compared to the typical flow time scale $T_{\mathrm{a}}$, where $T_{\mathrm{a}}=D / U_{\text {mean }}=8.9 \mathrm{~s} .{ }^{20}$ In other words, the particle Stokes number is $S t=T_{\mathrm{p}} / T_{\mathrm{a}} \ll 1$, meaning that the tracer particles are passively advected by the flow. The drift velocity due to buoyancy $U_{\mathrm{p}}=\left|\rho-\rho_{\mathrm{p}}\right| g d_{\mathrm{p}}^{2} /(18 \rho v)=3.1 \mu \mathrm{m} / \mathrm{s}$ is much less than the fluid velocity $U_{\text {mean }}=6.3 \mathrm{~mm} / \mathrm{s}$ and yields a typical deviation in a particle trajectory, i.e., $\Delta x_{\mathrm{p}}$ $\equiv U_{\mathrm{p}} T_{\mathrm{a}} \sim 0.2 \mathrm{~mm}$, during its residence within the test section. This is also considered negligible.

Four synchronized 12 bit CCD cameras (with a resolution of $1600 \times 1200$ pixels; MegaPlus ES2020, Kodak, United Kingdom) equipped with $50 \mathrm{~mm}$ lenses (type NMV50M1, Navitar, USA) are positioned and aligned in such a way that each camera views the test section from a different perspective. This configuration enables an efficient matching performance for the 3D PTV algorithm. Camera control, synchronization and data storage are achieved by a data acquisition system (DVCR5000, Iris Vision, The Netherlands). ${ }^{17}$ The non-commercial 3D PTV algorithm developed at ETH Zurich, Switzerland, ${ }^{21,22}$ which is capable of performing particle matching and trajectory construction based on a four camera configuration, is used in the current study. The calibration procedure is explained in Section III C 2.

In the study by Jilisen et al. ${ }^{17}$ the error in measurements is found to be around $10 \%-15 \%$. In the present study, the error is reduced significantly by a new method comprising (i) a correction procedure in post-processing of data where a low-pass filtering is employed for particle trajectories via fitting cubic spline to the segments of the trajectories (for details see Ref. 22) and (ii) a modified calibration procedure (Section III C 2).

\section{Modification in 3D PTV calibration}

The current study employs the 3D PTV algorithm developed at ETH Zurich for the determination of 3D particle

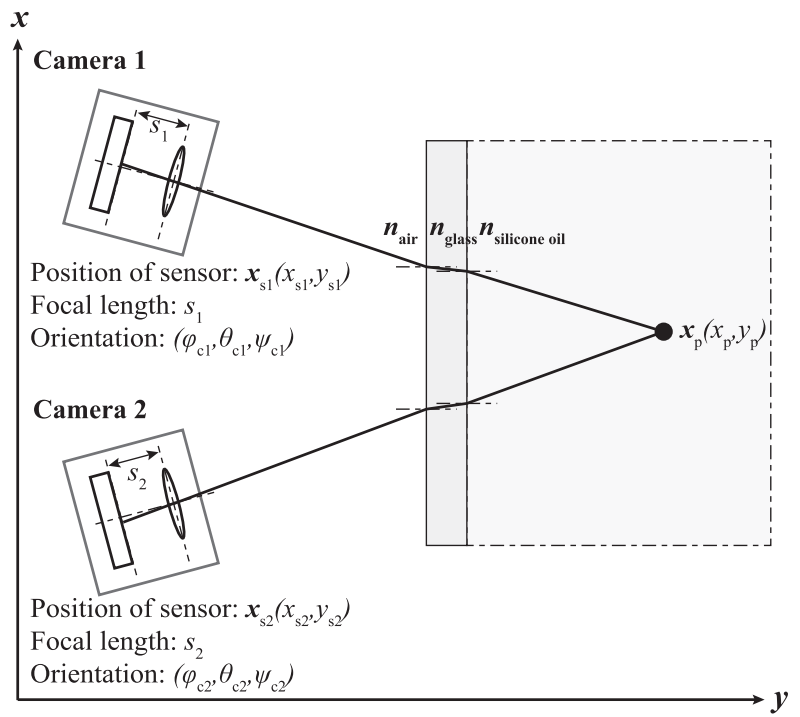

(a) coordinates and the reconstruction of the trajectories of the particles. This algorithm performs calibration and position calculations taking into account two flat interfaces between media with different refractive indices. It models only the presence of the cubic box (i.e., air-glass, glass-silicone oil) of the test section and ignores the cylinder walls (see Fig. 3(a)). This cylindrical wall causes further refraction of the light rays (see Fig. 3(b)). This additional optical effect should be taken into account in the calibration process for more accurate reconstruction of the particle positions and trajectories.

The modified calibration procedure is accomplished in three steps: (i) initial calibration by use of the original 3D PTV algorithm, (ii) production of synthetic calibration images, and (iii) compensation for the error that arises from the presence of the cylinder. In the first step the system is calibrated in order to obtain the mapping function between the camera coordinates and the physical coordinates. To this end, a calibration body (for details, see Ref. 17) is placed inside the test section that is filled with the working fluid in order to mimic the actual fluid conditions. The images of the calibration body are processed by the ETH algorithm to acquire the calibration matrix albeit with an error due to the missing cylindrical wall in the optical model. Therefore, in the second step artificial images of the calibration body are generated based on the optical configuration shown in Fig. 3(b). The calculations are made with the camera position and orientation information as estimated in the first step by the ETH algorithm. These initial values are then varied with fine steps iteratively in the new model with the objective of minimizing the disparity between the actual camera images and the artificial images of the calibration body. Consequently, a good match is achieved with an average disparity of two pixels in the marker positions. Subsequently, a correction factor is introduced to the particle positions to compensate for the calibration error. This is done by generating images of 5000 synthetic particles at known positions by the use of the improved model acquired in the second step. The ETH algorithm is then used with the artificial images to reconstruct

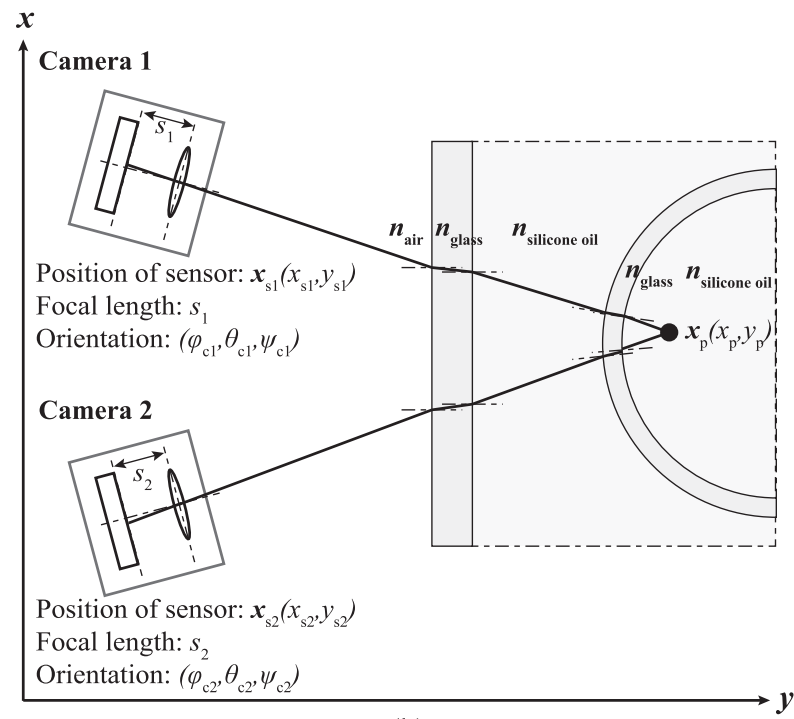

(b)

FIG. 3. (a) Optical configuration modeled by the ETH 3D PTV algorithm; (b) optical configuration of the actual experimental setup (note that this configuration is used in the artificial calibration image generation). 

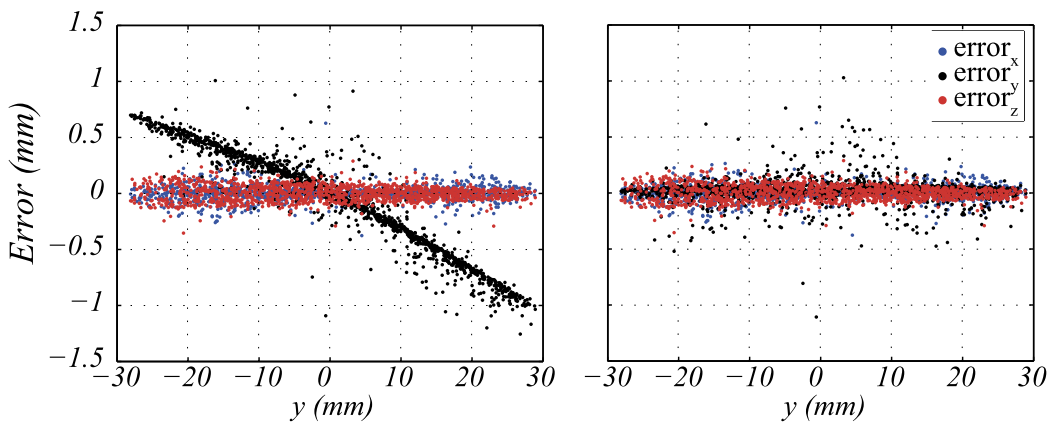

FIG. 4. The distribution of the error in the $x$ (blue), $y$ (black), and $z$ (red) coordinates of synthetic particles reconstructed by the ETH algorithm as a function of the $y$ positions of the particles before (first row) and after (second row) correction. the $3 \mathrm{D}$ particle positions. The error is calculated as the difference between the reconstructed and real positions of the synthetic particles in $x$-, $y$-, and $z$-directions, i.e., $\boldsymbol{\epsilon}=\boldsymbol{\epsilon}\left(\epsilon_{x}, \epsilon_{y}, \epsilon_{z}\right)$. The ETH algorithm with incomplete optical model (i.e., the optical configuration without the inner cylinder) performs well in the calculation of the $x$ and $z$ components of the particle positions; however, there is a significant systematic error in the calculation of the $y$-component, displaying an almost linear trend in the $y$ direction (depth direction of the camera configuration), as shown in Fig. 4. The relation between the $y$ position of the particles and the $y$ component of the error is used to derive an empirical correction factor for the corrected $y$ positions of the particles,

$$
y_{\text {cor }}=\left\{\begin{array}{ll}
y\left(1-\frac{1}{R}\right), & \text { for } y \geq 0 \\
y\left(1-0.68 \frac{1}{R}\right), & \text { for } y<0
\end{array},\right.
$$

where $R$ is the dimensionful radius of the tube $(R=28 \mathrm{~mm})$ and $y_{\text {cor }}$ is the $y$-component of the corrected particle positions. As a result of this correction, the overall error is decreased significantly showing random variations with respect to particle positions (similar as those for the $x$ - and $z$-direction) and no systematic deviation. This improves the calculation of the particle positions compared to the work of Jilisen et al., ${ }^{17}$ and the effect of the correction on the processed data (i.e., velocities) is discussed further in Section IV.

\section{3D laser-induced fluorescence}

The 3D laser induced fluorescence (3D LIF) measurements are performed in a cylindrical volume with diameter $D=56 \mathrm{~mm}$ and a height of $800 \mathrm{~mm}$, in which 14 mixing elements (i.e., 7 mixing periods) are held. The measurement volume is illuminated by a continuous argon-ion laser (Stabilite 2016, Spectra Physics, USA) at a wavelength of $488 \mathrm{~nm}$ and a laser sheet scanner (LaVision, Germany) (see Fig. 5). The fluorescent oil is a mixture of an oil-soluble fluorescent dye (Sudan Orange 183 by BASF AG, Ludwigshafen, Germany) and silicone oil with a dye concentration of $40 \mu \mathrm{g} / \mathrm{l}$ and a fluorescence wave length of $580 \mathrm{~nm}$. This allows the visualization of the concentration field after filtering the laser wavelength by use of a $570 \mathrm{~nm}$ high-pass optical filter (Thorlabs, Germany). The images of the concentration fields are recorded using a high speed 12 bit CMOS camera (with a resolution of $1024 \times 1024$ pixels; HighSpeedStar 5.1, LaVision, Germany) with its optical axis perpendicular to the laser sheet. The camera is equipped with a lens (AF Nikkor 28-70 mm, Nikon, Japan) at a focal length of $70 \mathrm{~mm}$. The aperture of the camera lens is set to the f-number $f \#=5.6$. An increase in f-number results in an increase in the depth of field while causing a decrease in the amount of light recorded, which results in a decrease in the signal-to-noise ratio of the measurements. In order to acquire in-focus images of the scalar patterns at a relatively high signal-to-noise ratio, the measurements are repeated by focusing the camera at two different planes along the diameter of the tube. The focal plane is positioned in the middle of the near half-diameter in the first run and then it is moved to the middle of the far half-diameter for the second run. The field of view of the camera in the center plane of the tube is approximately $65 \times 65 \mathrm{~mm}$ and in order to acquire the concentration field throughout the test section the image acquisition is repeated at 23 positions in the axial direction. This is facilitated by a manually operated transversing device.

The synchronization between the illumination system and high-speed imaging system is performed by a signal generator (Stanford, USA) and a high-speed controller (LaVision, Germany). The signal generator produces a TTL signal at a frequency of $0.05 \mathrm{~Hz}$ that is input to the laser sheet scanner,

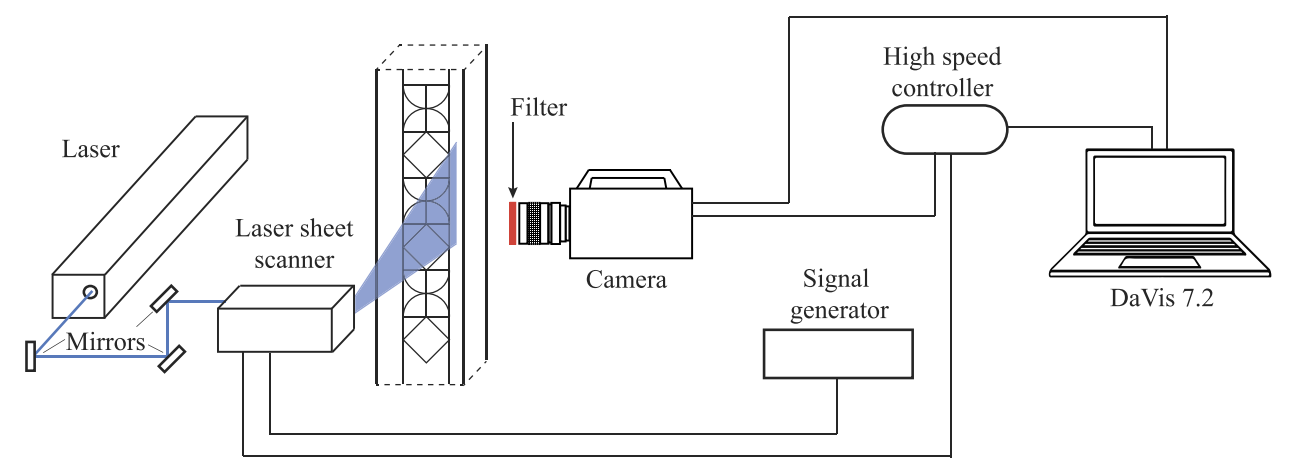

FIG. 5. A schematic sketch of the 3D LIF data-acquisition system. The system is composed of a continuous laser equipped with a laser sheet scanner device, a high-speed camera with a multifocal lens on which a high-pass filter is mounted, a signal generator by which the laser sheet scanner is activated, and a high speed controller for the synchronization of these devices. 
which results in a scanning period of $20 \mathrm{~s}$. The high-speed camera is configured to capture images at a recording frequency of $50 \mathrm{~Hz}$. The image acquisition is started by a trigger signal that is generated by the laser sheet scanner and received by the high-speed controller. This configuration brings in a total of 1000 images captured during one turn-around time of the laser scanner. Image acquisition and preprocessing are performed by the commercial software package Davis 7.2 (LaVision, Germany).

a. Calibration and corrections. The experiments aim at the (qualitative) investigation of the scalar patterns rather than a quantitative analysis of the concentration distribution. Hence, the scalar field is examined in terms of the recorded fluorescence intensity with the assumption that the camera has a linear response to fluorescence intensity. Nonetheless, for a better image quality, several corrections are applied. First, to improve the contrast in the concentration field, dark image calibration is performed by subtracting the dark images taken in a dark room (when the camera lens is covered) from the raw images. Second, the correction images captured at uniform concentration values (by running only the fluorescent oil in the test section) are analyzed to check the effect of the attenuation of the laser on the camera readings. The data analysis shows that the attenuation due to absorption of the dye is negligible. Finally, all images are resized to fit onto the same circular cross section according to their distance to the sensor. By use of the resized 2D images, the 3D concentration field is constructed and the downstream cross-sectional evolution of the scalar patterns is investigated.

\section{Data processing and analysis}

Data processing and analysis of scalar transport is based on the dynamic mode decomposition (DMD). This algorithm enables recovery of the eigenmode decomposition (Eq. (A1)) from data at discrete temporal/spatial levels. It assumes that the subsequent levels relate via a mapping,

$$
\boldsymbol{c}_{\mathrm{n}+1}=\mathcal{A} \boldsymbol{c}_{\mathrm{n}},
$$

where $\boldsymbol{c}_{\mathrm{n}}$ denotes the scalar field in discrete partitions in the domain at level $z_{\mathrm{n}}=n \Delta z$ and $\mathcal{A}$ is a linear operator that maps a scalar field $\boldsymbol{c}_{\mathrm{n}}$ to the consecutive one $\boldsymbol{c}_{\mathrm{n}+1}$. The eigenfunctioneigenvalue pairs of the mapping matrix $\mathcal{A}$ are the approximations for the eigenfunction-eigenvalue pairs $\left\{\varphi_{k}, \mu_{k}\right\}$ of the advection-diffusion operator $\mathcal{L}$. The details of the algorithm can be found in Refs. 23 and 24.

\section{PERFORMANCE ANALYSIS OF THE MODIFIED 3D PTV PROCEDURE}

In order to quantify the error in 3D PTV experiments, a comparative analysis between experimental and numerical velocity fields is performed for velocity components $u, v$ and $w$, and magnitude $\|\boldsymbol{u}\|$, for which more than 18000 data points are taken into account. The positions are taken from the experimental data set, and to obtain the corresponding numerical values a cubic interpolation fit is employed on the simulated data. The deviations in the experimental data are quantified via $\epsilon(\mathrm{u}) \equiv\left|\mathrm{u}_{\mathrm{n}}-\mathrm{u}_{\mathrm{e}}\right| /\left|\mathrm{u}_{\mathrm{n}, \max }\right|$ where u can be $u, v, w$ or $\|\boldsymbol{u}\|$
( $\mathrm{n}$ and e stand for numerical and experimental data, respectively). The associated statistics of the error analysis, i.e., the mean $\mu$, median $M$, and standard deviation $\sigma$ of the error, are given in Table I, where the numbers are given in percentage. In spite of the correction applied to the $y$ positions of the particles, the maximum error is still observed for the velocity component in the $y$-direction. This is mainly due to increased refraction effects around the mid-plane of the cylinder close to the walls (i.e., $y \approx 0$ and $x= \pm R$ ), where the curvature with respect to the viewing direction increases considerably, resulting in elevated error values at these locations. These localized high errors result in relatively high standard deviation and mean error values for the $v$ component of the velocity. Overall, the error is found to be between 3\% and 7\% (which was around $10 \%-15 \%$ for the old algorithm ${ }^{17}$ ) which implies that the modified calibration procedure and the low-pass filtering in the particle tracks result in an improvement in postprocessing; hence, there is an overall good agreement between the numerical and the experimental results.

Figure 6 shows a sample of experimental and numerical trajectories, passing through the same position at the inlet of the mixer. The deviation between the trajectories is quantified by $\epsilon_{2}(t)=\sqrt{(\Delta x(t))^{2}+(\Delta y(t))^{2}+(\Delta z(t))^{2}}$, where $\Delta$ indicates the difference between experimental and numerical positions. Further analysis shows that the deviation increases in time and the maximum deviation is found to be $0.89 \mathrm{~mm}$. This is likely due to the imperfect alignment and positioning of the mixing elements. Nevertheless, the trajectories show a very good qualitative agreement.

\section{VELOCITY FIELD ANALYSIS}

\section{A. Periodicity of the flow field}

In fluid processing industries, inline mixers constitute a part of a piping system and can be placed at any location regardless of local flow conditions. This alters the inlet conditions of the flow field inside the mixer (i.e., they deviate from Poiseuille flow). However, the flow quickly settles on a periodic state, regardless of inlet conditions, which is demonstrated below.

In order to determine the earliest mixing element in which the flow has become periodic again, independent of inlet conditions, a comparative analysis is conducted. A mixer with 2 elements ( 1 period) is simulated with periodic boundary conditions at the inlet/outlet at $R e=3.5$ and this case is set as the benchmark case for comparison. Moreover, a mixer

TABLE I. Statistics of the error analysis for velocity components $u, v, w$, and magnitude $\|\boldsymbol{u}\|$. The error is quantified via $\epsilon(\mathrm{u}) \equiv\left|\mathrm{u}_{\mathrm{n}}-\mathrm{u}_{\mathrm{e}}\right| /\left|\mathrm{u}_{\mathrm{n}, \max }\right|$ where $\mathrm{u}$ can be $u, v, w$, or $\|\boldsymbol{u}\|$ (n and e stand for numerical and experimental data, respectively). The numbers are in percentage.

\begin{tabular}{lccc}
\hline \hline $\begin{array}{l}\text { Velocity components } \\
\text { and magnitude }\end{array}$ & $\begin{array}{c}\sigma_{\epsilon} \\
\text { (Standard deviation) }\end{array}$ & $\begin{array}{c}\mu_{\epsilon} \\
\text { (Mean) }\end{array}$ & $\begin{array}{c}M_{\epsilon} \\
\text { (Median) }\end{array}$ \\
\hline$u$ & 3.34 & 0.18 & 0.16 \\
$v$ & 6.76 & 1.21 & 0.24 \\
$w$ & 3.69 & 0.13 & 0.17 \\
$\|\boldsymbol{u}\|$ & 6.50 & 1.06 & 0.27 \\
\hline \hline
\end{tabular}



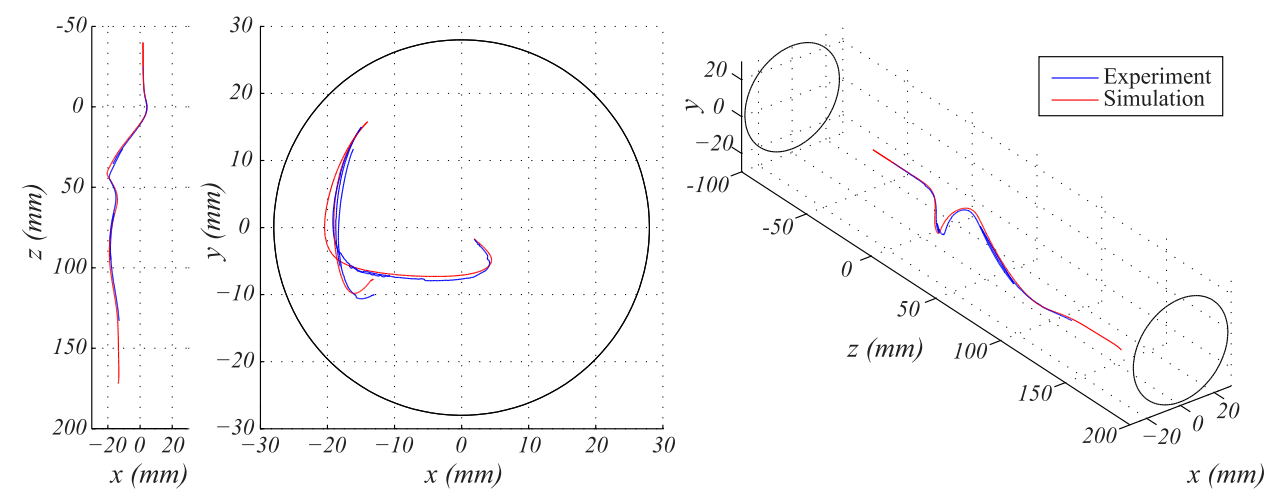

FIG. 6. Comparison of experimental (blue) and numerical (red) particle trajectories. From left to right: side view (normal to $x z$-plane), top view (normal to $x y$-plane), and isometric view. with 8 elements (4 periods) is simulated with three different inlet conditions, i.e., (i) open inlet with Poiseuille flow, (ii) partly blocked inlet (square blockage in center), and (iii) partly blocked inlet with a quarter circle blockage. A pressure boundary condition is applied at the outlet, and the mean flow (thus pressure gradient) is chosen such that once again $R e=3.5$. For the benchmark case, an arbitrary axial line segment connecting the points $\mathbf{r}_{\mathrm{p} \text {,initial }}=(15,15,0)$ (in $\mathrm{mm}$ ) and $\mathbf{r}_{\mathrm{p}, \text { final }}=(15,15,112)$ (in $\mathrm{mm}$ ), the length of which is equal to the length of one period, is defined as a line probe. The velocity magnitude distribution on this line is set as the benchmark for the comparison. Then, a similar line (this time the line segment is connecting the points $\mathbf{r}_{\mathrm{p} \text {,initial }}=(15,15,0)$ and $\mathbf{r}_{\mathrm{p} \text {,final }}=(15,15,336)$, the length of which is equal to the length of three periods) is defined as a line probe for the cases with non-periodic boundary conditions. The velocity magnitude distributions of all cases, i.e., the benchmark and the non-periodic cases, on these lines are plotted in Fig. 7 (left), which shows that any disturbance at the inlet vanishes within the second element and all cases converge to the benchmark case. Therefore, it is plausible to conclude that the flow field at $R e=3.5$ is basically independent of inlet conditions-an important fundamental characteristic of the flow-and quickly settles for one and the same periodic state. Here, this happens already within the second element of the first period, meaning that the periodic flow can be assumed from the second period onwards.

In order to quantify the global error, now sampling a volume instead of evaluating the velocity along a line segment, in the non-periodic cases described above (this time there are two more cases which are acquired experimentally: open inlet with Poiseuille flow and partly blocked inlet with a square in the center), a comparative analysis is performed between the benchmark case (the numerical case with periodic boundary conditions) and the non-periodic cases (experimental and numerical). Only the second periods of the non-periodic cases are used in the analysis. The analysis is performed on 18000 data points per case, where the positions of the data points are taken from the experimental data set. The corresponding data for the numerical simulations are calculated by a cubic interpolation. The error is quantified via $\epsilon(\mathrm{u}) \equiv\left|\mathrm{u}_{b}-\mathrm{u}_{c}\right| /\left|\mathrm{u}_{b, \max }\right|$ where $\mathrm{u}$ can be the velocity components $u, v, w$ and magnitude $\|\boldsymbol{u}\|$. Here, $b$ and $c$ stand for the benchmark case and any other case, respectively. Figure 7 (right) shows the standard deviation of the error distribution for the above-mentioned cases. The deviations in the numerical cases are below $0.1 \%$ which is equivalent to the relative tolerance of the flow solver (see Section III A). Moreover, the experimental statistics are consistent with the error estimated for 3D PTV in Section III C 1 and errors are typically less than $7 \%$. This analysis supports the periodic flow assumption from the second period onwards and reveals the agreement between the experiments and the numerical simulations.

\section{B. Velocity field}

This section analyzes the flow field of the Quatro mixer at $R e=3.5$ experimentally and numerically. A similar analysis is
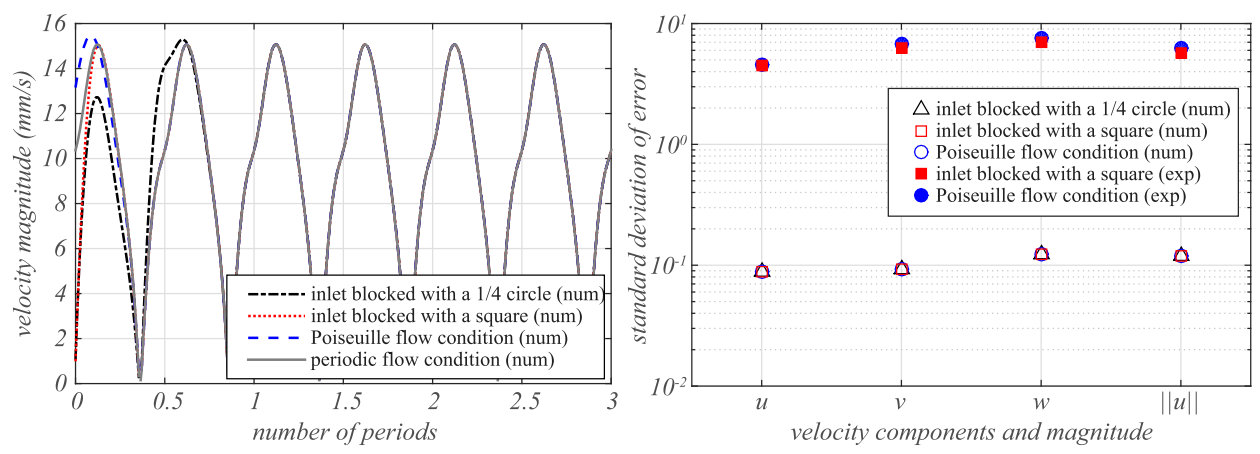

FIG. 7. (Left) The velocity magnitude distributions on an arbitrarily chosen line ( $x=0.015 \mathrm{~m}, y=0.015 \mathrm{~m}$ line) for the cases with different boundary conditions: (i) quarter circle blockage, (ii) square blockage, (iii) Poiseuille flow conditions at the inlet and pressure boundary condition at the outlet, and (iv) periodic boundary conditions at the inlet/outlet. Only the numerical simulations are shown. For all cases $R e=3.5$. (Right) The standard deviation of the error in $u, v$, and $w$ components of the velocity and the velocity magnitude $\|\boldsymbol{u}\|$. Filled markers: experiments, open markers: numerical simulations. The values are in percentage. 

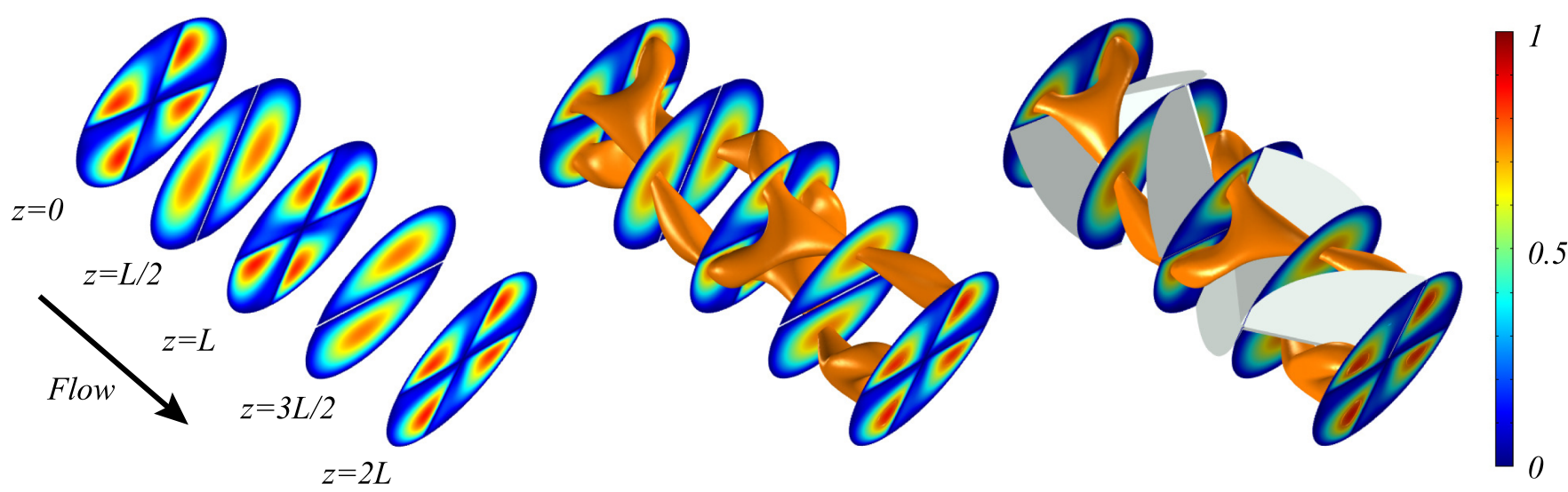

FIG. 8. Cross-sectional evolution of numerical dimensionless velocity magnitude along a period (which is composed of two mixing elements) of the Quatro mixer at $R e=3.5$ : (left) $2 \mathrm{D}$ plots of non-dimensional velocity magnitude at five axial positions $(z=0, L / 2, L, 3 L / 2,2 L$ ) along the mixer, (middle) $3 \mathrm{D}$ isosurface for the non-dimensional velocity magnitude $\|\boldsymbol{u}\|=0.75$, and (right) the orientation of the blades. All values are non-dimensionalized with the maximum of velocity magnitude $\|\boldsymbol{u}\|_{\max }=0.022 \mathrm{~m} / \mathrm{s}$.

given in Ref. 17. The main difference between the two studies is the flow regime (see Section III B).

In order to illustrate the flow in the mixing elements, 2D plots of non-dimensional velocity magnitude at five axial positions along a periodic segment of the mixer, a 3D isosurface of the velocity magnitude, and the orientation of the mixing elements are shown in Fig. 8. As mentioned earlier, the second element of a periodic segment is the reflected and rotated version of the first one. The effect of this configuration is clearly visible in the cross-sectional flow fields. The flow field pattern at $z=L$ is essentially a mirrored version of that at $z=0$, while the pattern at $z=3 L / 2$ is a rotated form of the flow field at $z$ $=L / 2$. The velocity pattern repeats itself at $z=2 L$ in accordance with the periodic configuration of the geometry. The 3D isosurface also has a repeating pattern. Each segment of the isosurface (the length of a segment is $L / 2$ ) is a reflected version of the preceding one.

Figure 9 shows 2D plots of the velocity components $u$, $v$, and $w$ with the transversal velocity vectors $\boldsymbol{u}_{t}=(u, v)$ at five axial locations. The effect of the mixer configuration is also visible in the plots of velocity components. In general, the axial flow component is dominant, whereas the transversal flow components $u$ and $v$ are marginal, particularly at the inlet/outlet regions. Stronger transversal velocity components at the mid-plane (compared to the inlet/outlet planes) indicate that the flow has a higher circulation in this region.

Figure 10 shows the contour plots of the velocity magnitude at the inlet and the mid-plane of the first element obtained via 3D PTV experiments and computed numerically. Despite the quantitative differences, similar flow structures emerge in both cases. There are two main reasons for the poorer data quality in experimental plots: the optical obstruction by the edges of the mixing elements and very few particle tracks near the walls. The effect of the former is well visible in Fig. 10(a). As the cameras are positioned in the region where $y>0$ (less optical obstruction in this region), the top half of the plot $(y>0)$ shows a better agreement compared to the bottom half $(y<0)$ of the plot. The effect of the latter (limited number of particle tracks near the walls) can also be clearly seen in the experimental results. Hence, the determination of the velocity field in these regions is possible only by the interpolation of the neighboring data points. This explains why the zero-velocity regions do not appear in the results even though they physically exist in the experiments. However, overall, the comparison reveals a good agreement between the experiments and the simulations.
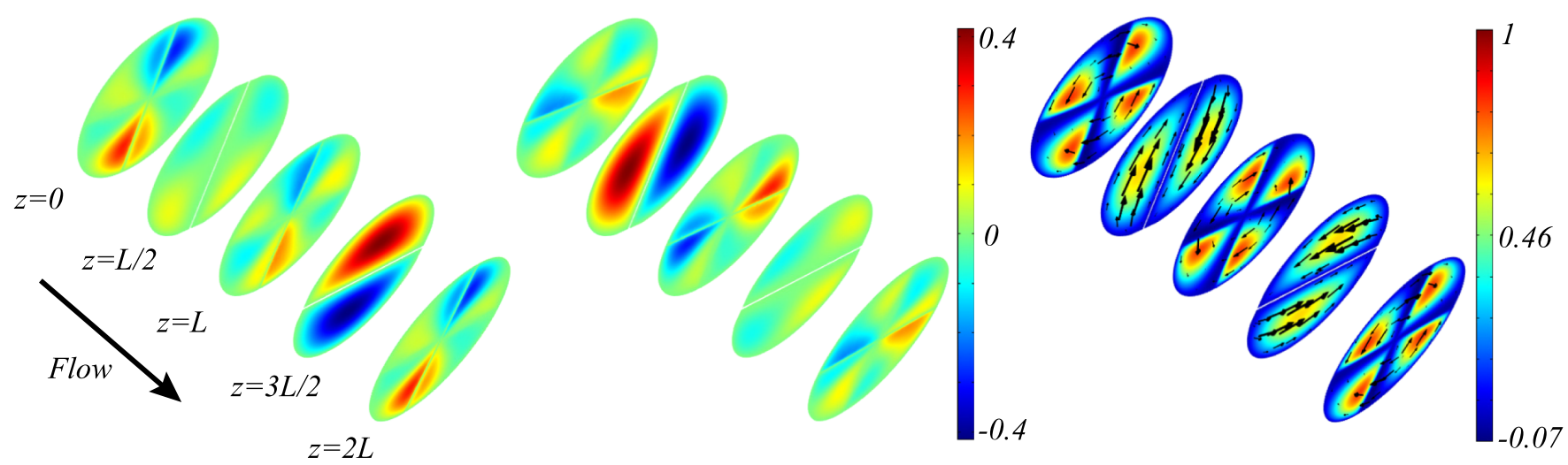

FIG. 9. Cross-sectional evolution of numerical dimensionless velocity field components at five axial positions $(z=0, L / 2, L, 3 L / 2,2 L)$ along the Quatro mixer (which is composed of two mixing elements) at $R e=3.5$ : (left) $u$ component, (middle) $v$ component, and (right) $w$ component with the transversal velocity vectors $\boldsymbol{u}_{t}=(u, v)$. All values are non-dimensionalized with the maximum of velocity magnitude $\|\boldsymbol{u}\|_{\max }=0.022 \mathrm{~m} / \mathrm{s}$. 

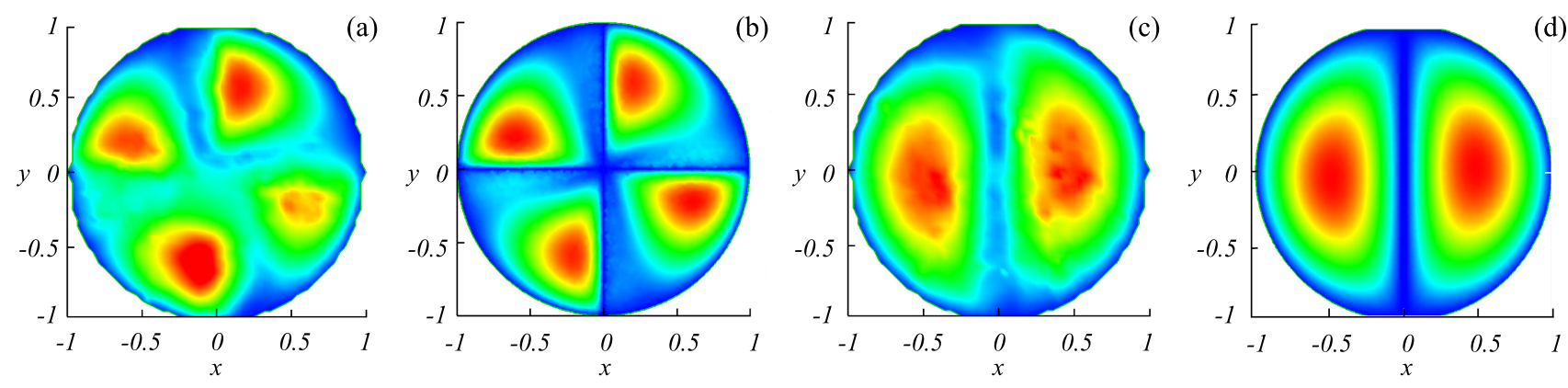

FIG. 10. Dimensionless velocity magnitude at the inlet ((a) and (b)) and the mid-plane ((c) and (d)) acquired experimentally ((a) and (c)) and numerically ((b) and (d)) at $R e=3.5$. Color code: blue (0)-green (0.5)-red (1).

\section{Streamlines and Poincaré section}

This section addresses the investigation of the transport properties of the Quatro mixer flow from a Lagrangian point of view, which enables the identification of coherent structures that form in the 3D streamline portrait as shown in Fig. 11. The streamlines in Fig. 11(a) are obtained experimentally by 3D PTV. In order to illustrate the mixing principle of the Quatro mixer, the streamlines are colored differently based on their location with respect to the first element of the segment: streamlines that enter via one (i.e., the upper) side of the element are colored in green, whereas those that enter via the other (lower) side are in red. These green and red streams are divided into two by the leading edge of the second mixing element. Concurrently, green and red streams merge on both sides of the second element. This process repeats itself in the subsequent mixing segments and is thus intended to lead to mixing of incoming fluid streams.

The streamline clusters in Fig. 11(a) demonstrate only the mixing principle (i.e., division and confluence of the flow stream by the internal elements) of the Quatro mixer. However, this is itself not a guarantee for efficient mixing. The mixing characteristics can be determined from the Lagrangian topology of the flow field, which enables isolation of mixing and non-mixing regions. To this end long-time behavior of fluid parcel trajectories should be obtained. In the current study, this can only be achieved numerically since an experimental facility with a large number of segments is not feasible. In this context, the long-time behavior of the fluid parcel trajectories, which are acquired numerically, are analyzed via Poincare sectioning, where intersections of the initially tagged fluid parcel trajectories with cross-sectional planes (which are perpendicular to the z-axis and positioned in a way that axial distance between any two consecutive planes is $2 L$, i.e., the axial length of a periodic segment of the Quatro mixer) are collected in a single cross-sectional plane. This Poincaré sectioning is a common technique for examining inline mixers. ${ }^{6,9,11,13-15}$

The Poincaré section of the Quatro mixer under current flow conditions (and $R e=0.5$ ) is obtained by introducing 40 randomly distributed tracer particles at the mid-plane of the second mixing element of a periodic segment of the Quatro mixer. This is for consistency with the scalar field analysis in Section VI. Thisparticular axial position is chosen for practical reasons discussed in Section VI and does not have any influence on the interpretation of the topological structure of the flow field. These particles are tracked numerically by using the volume-preserving integration scheme following Ref. 11, which yields the Poincaré section shown in Fig. 11(b). The Poincaré section reveals globally chaotic advection without the emergence of any non-mixed regions, conclusively demonstrating good mixing by the Quatro mixer. Note that the Reynolds number of the flow field used for Poincare sectioning is $R e=0.5$ for consistency with the scalar field analysis given below.

\section{ADVECTIVE-DIFFUSIVE SCALAR TRANSPORT ANALYSIS}

This section presents a comparative analysis between experimental observations and numerical simulations on the
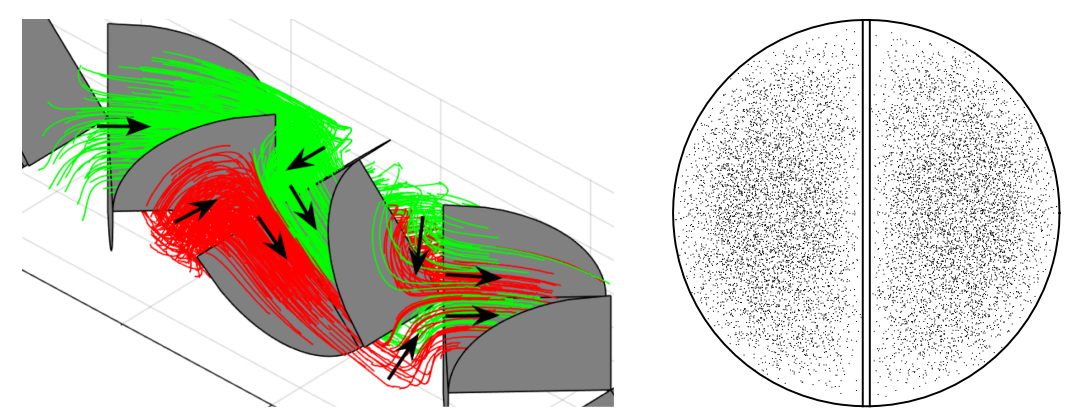

(a)
FIG. 11. (a) Measured 3-D streamlines in a segment (with three mixing elements) of the Quatro mixer for Re $=3.5$, (b) the Poincaré section of the numerical Quatro mixer flow for $R e=0.5$. The axial position of the cross section is $z=3 L / 2$ and the vertical lines represent the central chevron-shaped blade of the Quatro mixer. 
cross-sectional evolution of the 3D scalar field in the Quatro mixer, the configuration of which is given in Section II. The experiments concern the scalar transport in the advective limit where Péclet number is extremely high (yet not infinite). However, in the numerical simulations accurate computations are possible only up to $P e=1000$ due to the restriction imposed by the numerical resolution. We have discussed in Ref. 25 that the diffusion becomes relevant only for spatial features below a certain minimum length scale, i.e., the universal minimum length scale $\delta$ which is determined by the diffusion coefficient of the scalar, whereas for the features above $\delta$ diffusion is irrelevant. Hence, despite difference in $P e$, we can nonetheless perform a comparative analysis for larger features in the scalar patterns, which correspond to the patterns in the earlier cross sections of the evolution.

\section{A. Eigenmode analysis}

In this section, the advective-diffusive scalar transport in the Quatro mixer is investigated by an eigenmode analysis (the Appendix), and DMD (Section III D) is applied on the numerical scalar field data to reveal the scalar distribution that dominates the downstream evolution. The numerical model of the mixer consists of 10 periodic segments. In experimental data processing, the $2 \mathrm{D}$ cross-sectional scalar distributions representing the downstream evolution are extracted from the 3D data set at the mid-plane of every second mixer element rather than the inlet/outlet planes of the periodic elements. This is due to the fact that in the experiments the edges of the mixer elements deteriorate the image quality, and the selected cross sections are the ones which suffer the least from the disturbance by the edges of the elements. The same procedure is also applied to the numerical scalar fields to be consistent.

Figure 12 shows the numerical (top, color) and experimental (bottom, gray scale) downstream evolution of an initially non-uniform scalar field in the Quatro mixer. The inflow is composed of two scalar concentration levels (i.e., $\left.C\right|_{\text {inlet }}=1$ for $y>0$ and $\left.C\right|_{\text {inlet }}=0$ for $y<0$ ). As the flow passes through the mixing elements, initially segregated concentration fields break into smaller-scale structures by the effect of stretching and cutting processes. Diffusion, in the case of $P e=1000$, smoothes the high-gradient regions. Here, the downstream evolution is dynamically similar to the temporal evolution in 2D time-periodic systems: the initially segregated concentration field transforms into persistent patterns and evolves self-similarly with decreasing variance, as shown in Fig. 12. Comparison between experimental and numerical scalar fields
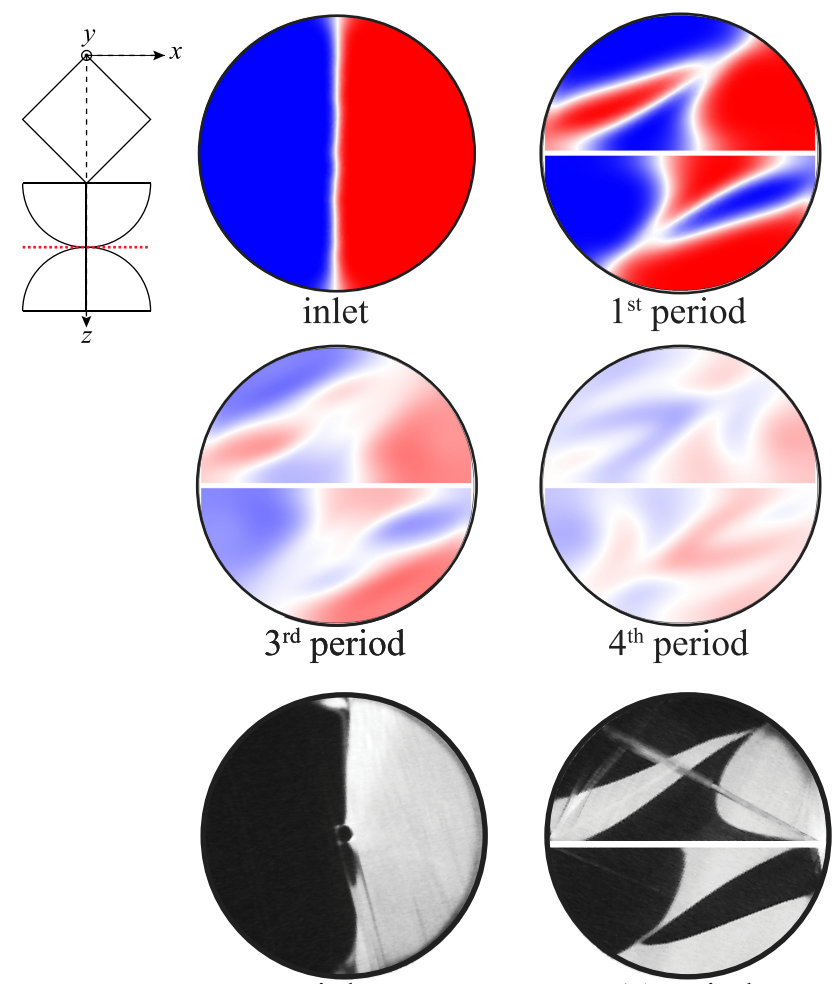

$1^{\text {st }}$ period
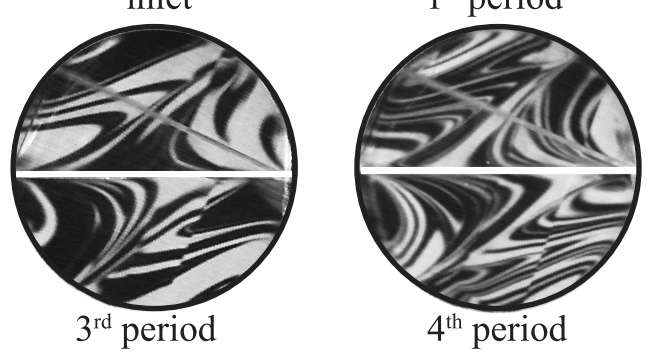
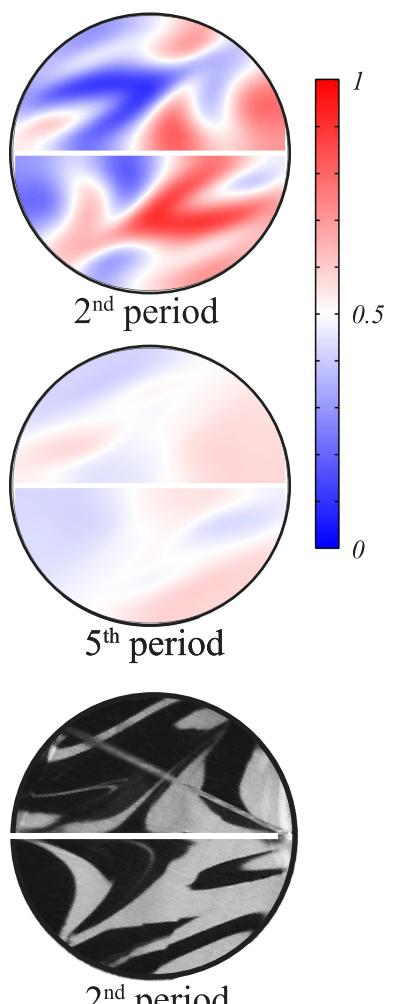

$2^{\text {nd }}$ period

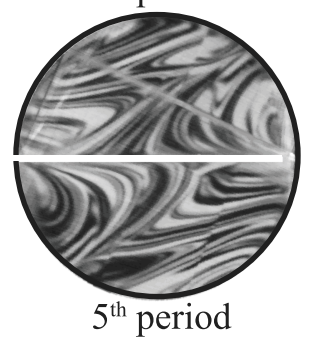

FIG. 12. Cross-sectional contour plots of numerical (colored) and experimental (gray scale) scalar distributions. The color range from 0 to 1 (dimensionless scalar field) is indicated from blue to red in the numerical results and black to white in the experimental results. The patterns are plotted for the dimensionless periods from 0 to 5 . $R e=0.5$ and $P e=1000$ in the numerical simulations whereas $R e=0.5$ and $P e \approx$ $\infty$ in the experiments. The red dashed line on the leftmost figure indicates the cross-sectional plane that the data are extracted. 
reveals that the overall patterns (especially for the earlier periods) agree well despite significant difference in $P e$. This is due to the fact that diffusion is negligible at these length scales, i.e., larger structures are insensitive to diffusion. The primary difference between experimental and numerical evolutions is that the experimental evolution continues to develop into ever finer structures while the simulated evolution very quickly becomes (nearly) homogeneous. This is due to the substantial difference in $P e$ and thus the far greater dominance of advection in the experimental case. Moreover, the experimental pattern clearly reveals a continuous process of stretching and cutting of material, which is an experimental evidence of chaotic advection. Thus, the pattern gradually outlines the unstable manifold that determines the chaotic advection. Finer structures emerging in the experiments also indicate that the experiments in fact outperform the simulations by revealing far greater detail.

On the basis of symmetry in the geometry and the flow field, there exists a clear anti-symmetry in cross-sectional scalar patterns, which is shown in Fig. 13. This anti-symmetry is very accurately present in the numerical results, yet it is less pronounced in the experiments due to imperfections in the facility. The symmetry comparison is only shown for the first two patterns of the evolution, since for higher $n$ values the comparisons are not very clear anymore due to the very fine structures in the experimental scalar fields.

The normalized numerical scalar fields in Fig. 14 reveal period- 2 behavior, implying that the evolution is dominated by period- 2 eigenmodes $\varphi_{1}(x, y)$ and $\varphi_{2}(x, y)$. The corresponding eigenvalues are of the form $\lambda_{j+1}=|\lambda| e^{2 \pi i j / p}$, where $j=0,1$ and the periodicity $p$ is 2 . Here, the magnitude of the exponential decay rate $|\lambda|=e^{\sigma}$ with $\sigma=\sigma_{1}=\sigma_{2}<0$. This leads to period-2 eigenvalues which can be written as $\mu_{1}=\sigma$ and $\mu_{2}=\sigma+i \pi$ and implies that both eigenmodes $\varphi_{1}(x, y)$ and $\varphi_{2}(x, y)$ decay with the same rate $e^{\sigma}$. The imaginary component of $\mu_{2}$, that is $\omega_{2}=\pi$, acts as an identity operator for $\varphi_{2}(x, y)$ for time spans of 2 periods (i.e., $\left.e^{\pi i n}=(-1)^{n}\right)$. Hence, the evolution of the concentration field can be approximated by the reduced expansion

$$
C(x, y, n Z)=\sum_{k=1}^{2} \gamma_{k} \varphi_{k}(x, y) e^{\mu_{k} n Z}+C_{\infty},
$$

where $C_{\infty}=0.5$ is the homogeneous final state.

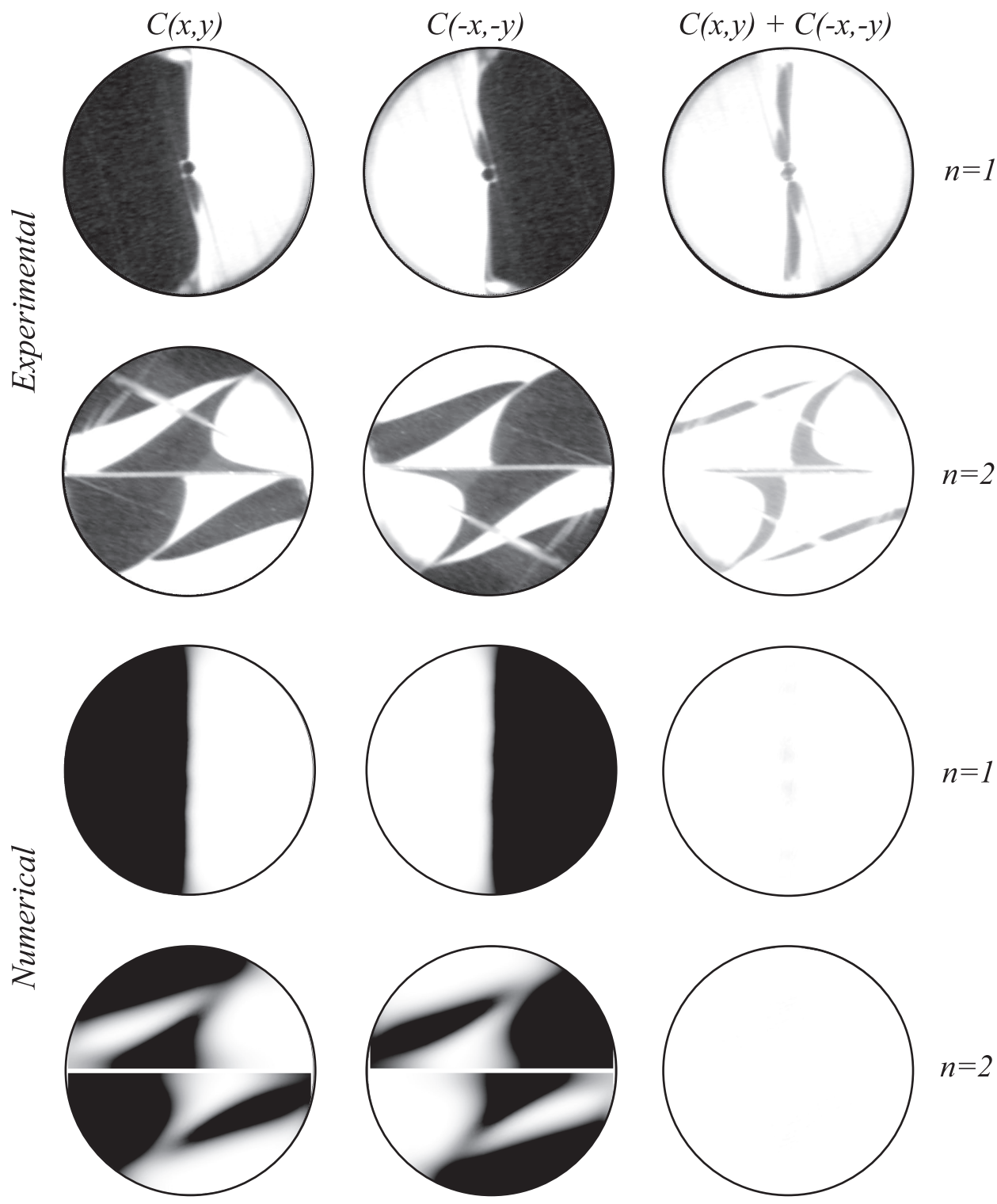

FIG. 13. Anti-symmetry in the scalar field for the first two patterns of the evolution, where $n=1,2$. First two rows: experiments, bottom two rows: numerical results. 

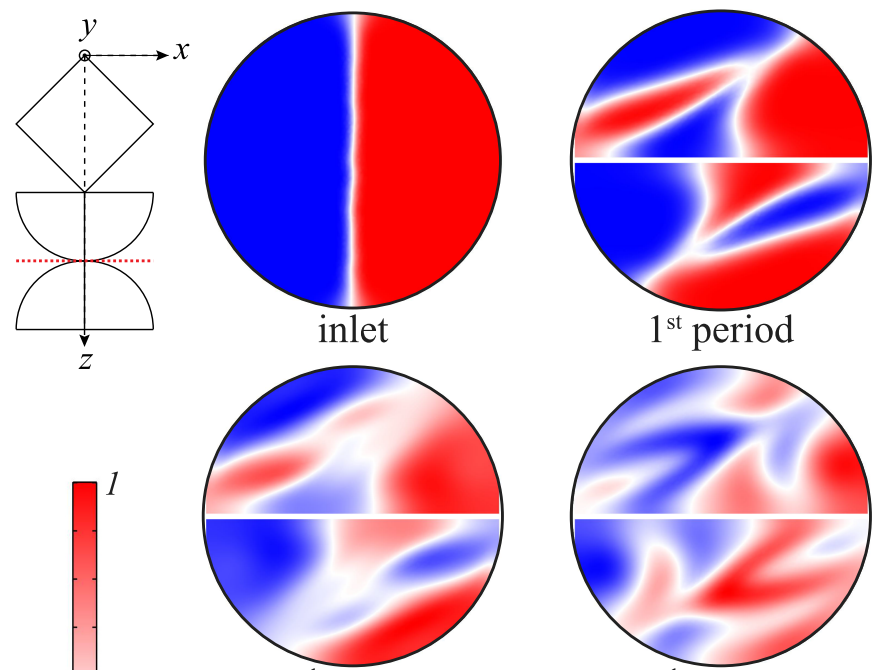

0.5

$3^{\text {rd }}$ period
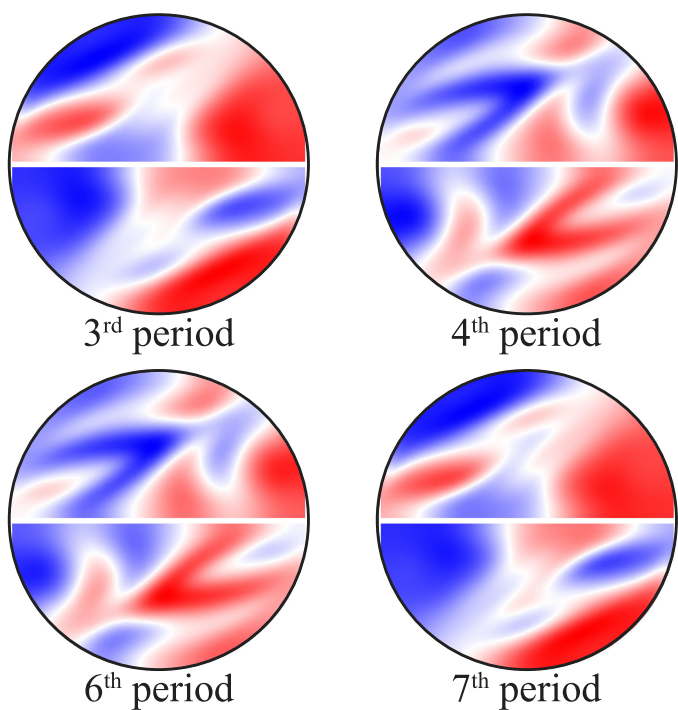

$4^{\text {th }}$ period
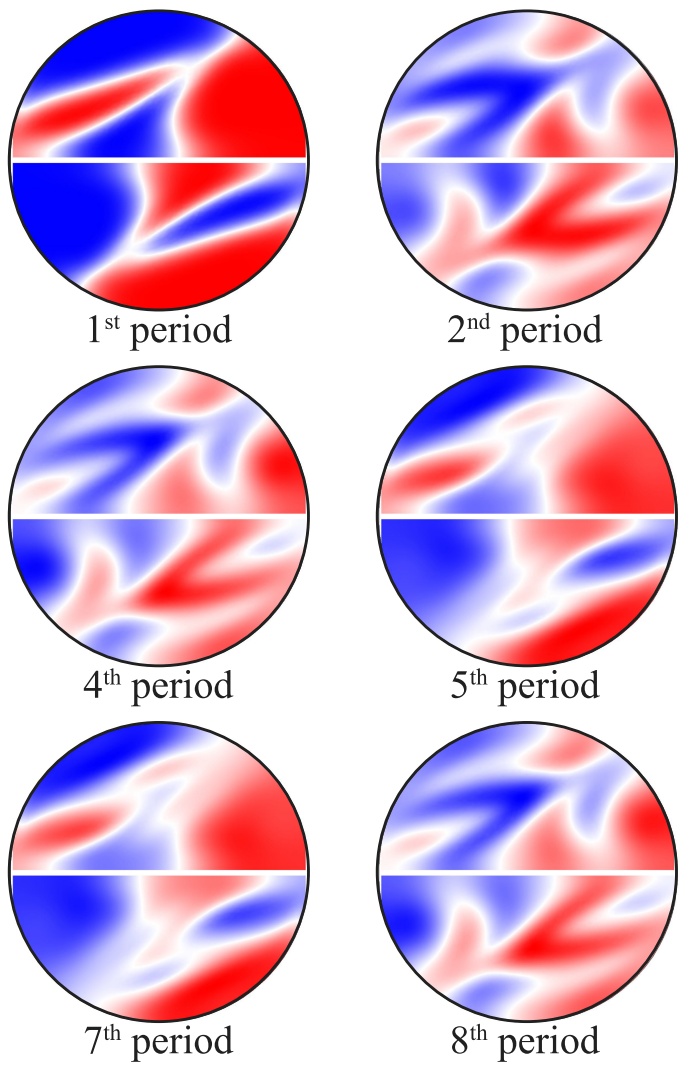

$5^{\text {th }}$ period

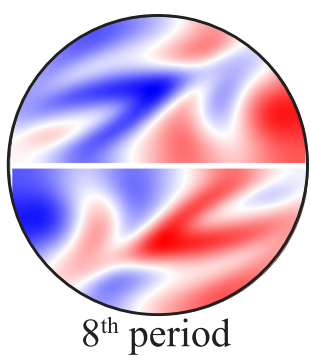

$8^{\text {th }}$ period
FIG. 14. The normalized evolution of the numerical concentration field (each contour plot is normalized by its own maximum and minimum values). The color range from 0 to 1 (dimensionless scalar field) is indicated from blue to red. The patterns are plotted for the dimensionless periods from 0 to 8 . Re $=0.5$ and $P e=1000$. The red dashed line on the leftmost figure indicates the cross-sectional plane that the data are extracted.
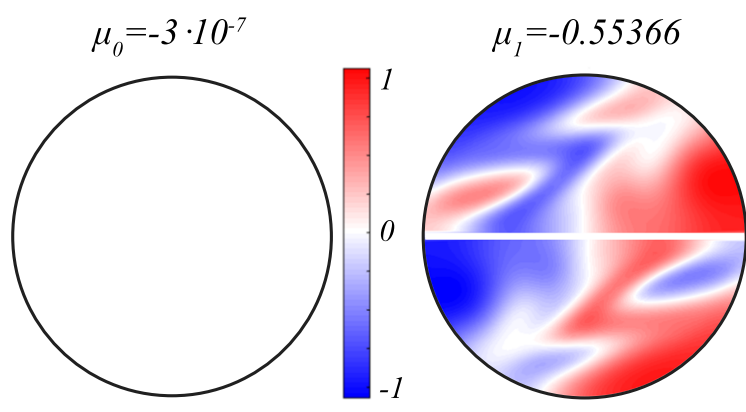

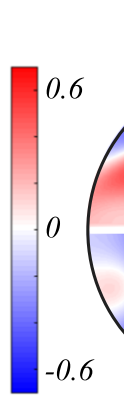

$\mu_{2}=-0.55622$

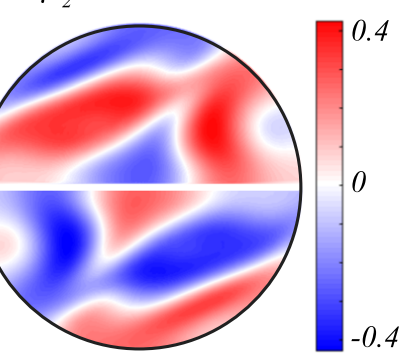

FIG. 15. The first three dominant eigenmodes of concentration field evolution of the numerical simulation for the case $P e=1000$ and $R e=0.5$. DMD is applied on the numerical data set to obtain the dominant eigenmodes.
Eigenmode decomposition (Eq. (8)) is verified by performance of DMD on simulated data. This gives the decomposition as shown in Fig. 15. The eigenvalues calculated by DMD are in accordance with the theory: the real part of the eigenvalues is the same (i.e., $\sigma_{1}=\sigma_{2}=\sigma \approx-0.55$ ) and the imaginary parts are $w_{1}=0$ and $w_{2}=\pi$ as given above.

The evolution is dominated by the eigenmodes shown in Fig. 15 meaning that the linear combination of these two eigenmodes gives the repeating patterns of the evolution (Fig. 14): for even values of $n$, the repeating pattern is given by $\varphi_{1}+\varphi_{2}$ (Fig. 16, left) and for odd values of $n$, the pattern is calculated as $\varphi_{1}-\varphi_{2}$ (Fig. 16, right).

\section{B. 3D Lagrangian transport}

In addition to the eigenmode analysis, experimental 2D cross-sectional scalar fields are analyzed to investigate the behavior of material fluid regions and the scalar quantities
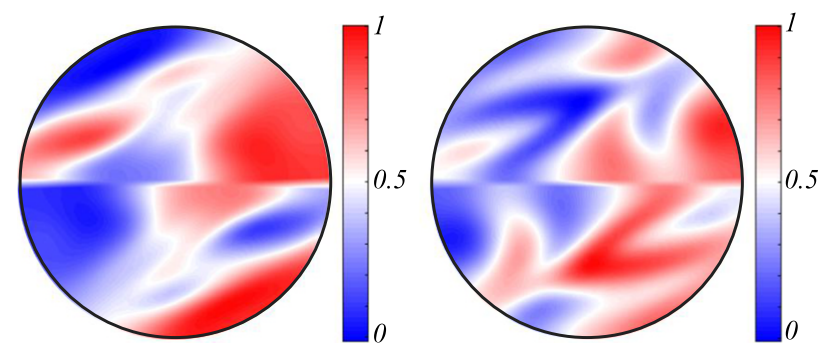

FIG. 16. Linear combination of the dominant eigenmodes: $\varphi_{1}+\varphi_{2}$ (left), $\varphi_{1}-\varphi_{2}$ (right).

they contain under the effect of stretching and folding mechanisms. The results presented in this section are obtained from 3D LIF measurements. In order to enhance the contrast in the scalar fields and facilitate easier interpretation of the results, the images are processed by sharpening the interface lines between white and black fluids and eliminating artifacts due to refractions and reflections. 

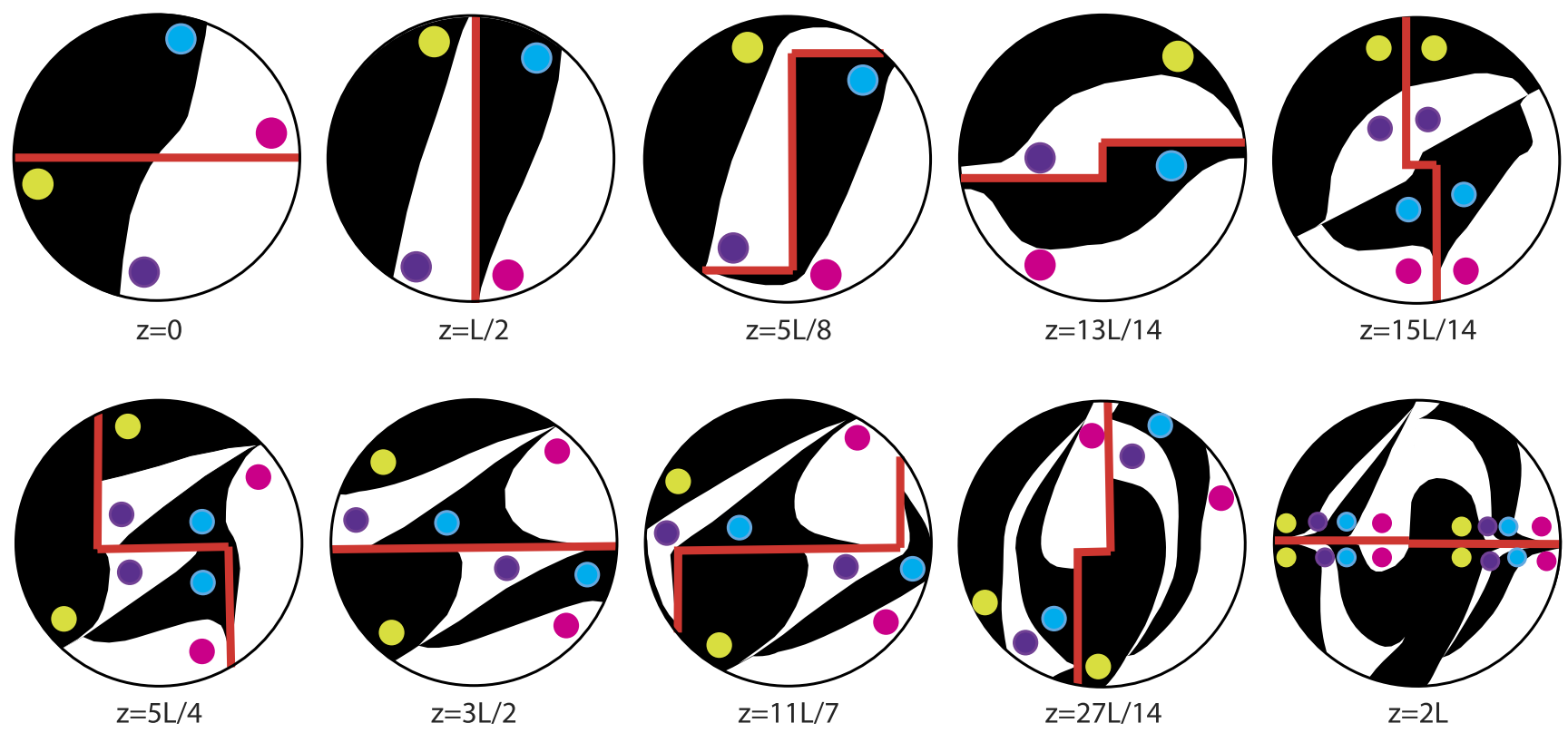

FIG. 17. Cross-sectional evolution of experimental scalar fields at 10 axial positions $(z=0, L / 2,5 L / 8,13 L / 14,15 L / 14,5 L / 4,3 L / 2,11 L / 7,27 L / 14$, and $2 L)$ along the first periodic segment of the Quatro mixer (which is composed of two mixing elements) at $R e=0.5$. The circles in color (yellow, pink, blue, and purple) are used to tag the evolving regions for a better understanding of the scalar evolution.

Figure 17 displays the evolution of the initially segregated scalar field from the Quatro mixer inlet to the outlet of the first periodic segment. The $2 \mathrm{D}$ cross sections are taken at several axial locations that are selected primarily on the basis of the image quality. At the inlet of the first mixing element $(z=0)$, the scalar field is comprised of four regions denoted by blue, pink, yellow, and purple circles. During the first quarter period, i.e., $0<z / L<L / 2$, the scalar fields are stretched in the $y$-direction, resulting in an increased interface between the white and black regions, thus enhancing mixing by molecular diffusion. The stretching occurs in the $x$-direction during the subsequent quarter of the period, i.e., $L / 2<z / L<L$, which can be seen from the cross section at $z=13 L / 14$. At the inlet of the second mixing element, i.e., $z=L$, the leading edge cuts the scalar field into two and thus produces eight segregated regions $(z=15 L / 14)$. This cutting process is succeeded by stretching until the end of the second element $(z=27 L / 14)$, further increasing interface lengths between white and black regions and thus further enhancing molecular diffusion. The stretching and cutting procedure continues in the subsequent mixing elements and thus paves the way to an ever greater number of ever smaller scalar regions. This continuously increases the interfacial area between white and black regions and thereby promotes molecular diffusion. This progressive subdivision into ever smaller segments is clearly shown in Fig. 18, where 2D scalar fields are plotted at the axial positions of $z=L / 2$, $5 L / 2$, and $9 L / 2$. In addition to the stretching and cutting, merging also occurs after the onset of the third period (see blue-yellow circles at $z=9 L / 2$ ). This might be due to the strong deformation and high concentration gradients in the region of the merger. If, e.g., two black regions are separated by a narrow band of white fluid, then diffusion in fact causes black fluid to cross over between the black regions, thus merging them. Note that this is not in conflict with the assumption that the experiments concern the advective limit. Diffusion at some point always comes into play if the concentration gradients are high enough.

The progression of the scalar field shown in Fig. 12 as well as in Figs. 17 and 18 reveals that material fluid regions basically are continuously cut in half in a way similar to the well-known baker's map. This suggests exponential interface stretching and thus is a strong indication of chaotic advection. ${ }^{5}$ Hence, the measured scalar fields provide experimental evidence in support of the earlier claim based on the Poincaré

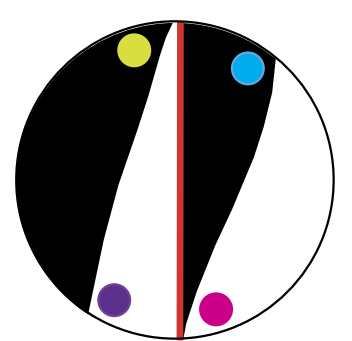

$\mathrm{z}=\mathrm{L} / 2$, mid plane of the 1 st element

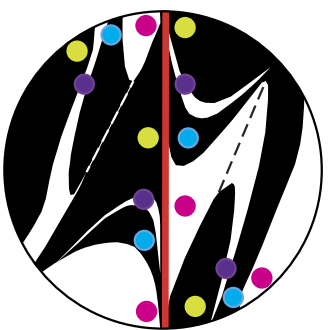

$\mathrm{z}=5 \mathrm{~L} / 2$, mid plane of the 3 rd element

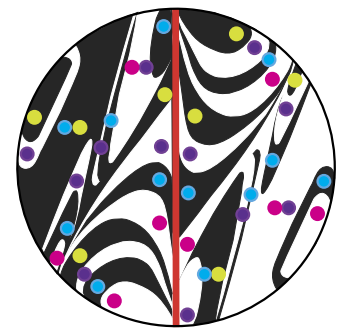

$\mathrm{z}=9 \mathrm{~L} / 2$, mid plane of the 5 th element
FIG. 18. Cross-sectional evolution of experimental scalar fields at 3 axial positions $(z=L / 2,5 L / 2$, and $9 L / 2$ ) which corresponds to the mid planes of the first elements of 1st, $2 \mathrm{nd}$, and 3rd periodic segments of the Quatro mixer. The circles in color (yellow, pink, blue, and purple) are used to tag the evolving regions for a better understanding of the scalar evolution. Right half of the rightmost image is enhanced by using anti-symmetry. 


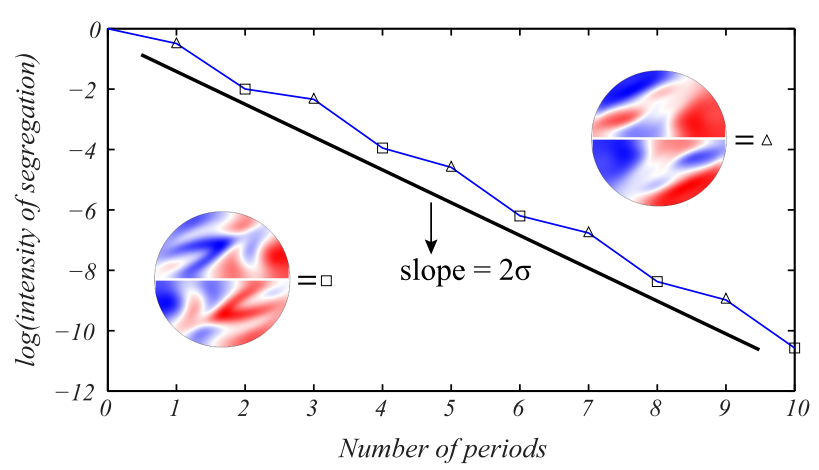

FIG. 19. Logarithmic decay of intensity of segregation for the cross-sectional evolution of the numerical concentration field at $P e=1000$ and $R e=0.5$.

section in Fig. 11(b) that the Quatro mixer indeed accomplishes chaotic advection and, as a consequence, efficient mixing.

\section{Quantification of mixing efficiency}

The intensity of segregation $I_{s}{ }^{26}$ is a statistical tool that quantifies the homogeneity of an initially segregated mixture, and it has already been employed in Refs. 27 and 25. The intensity of segregation is defined by

$$
I_{s}=\frac{\left\langle(I-\langle I\rangle)^{2}\right\rangle}{\langle I\rangle(1-\langle I\rangle)}
$$

where $I$ is the normalized corrected intensity of the scalar field $(0 \leq I \leq 1)$ and \langle\rangle the linear averaging operator. The intensity of segregation refers to the variation of the intensities in the mixture, and it tends to zero for perfect mixing.

The intensity of segregation, in this study, is calculated at the cross sections used for the eigenmode analysis. Figure 19 shows that the intensity of segregation decays exponentially similar to the decay of eigenmodes albeit at a different rate. The eigenmodes decay at a rate $\sigma=-0.55$, whereas the decay in the intensity of segregation is $2 \sigma=-1.10$. This is due to the square term in the definition of intensity of segregation (see Eq. (9)). The oscillatory behavior of the decay in the intensity of segregation also confirms the alternating behavior of the system between two repeating patterns.

\section{CONCLUSION}

This paper has shown that the application of an advanced calibration procedure together with a moving-average lowpass filter improved the accuracy of the 3D PTV measurements by decreasing the uncertainty in the measurement of particle positions from $10 \%$ to $15 \%{ }^{17}$ to the range of $3 \%-7 \%$. Moreover, this study has confirmed that the periodic flow assumption holds true for the flows inside spatially periodic inline mixers at low- $R e$.

The comparison between the numerical and experimental results shows that CFD modeling of 3D mixing is not yet powerful enough to reveal fine-scale structures in high- $P e$ transport. To this end, 3D experimental analysis as we demonstrate with the Quatro mixer study is required. The level of detail obtained experimentally exceeds that accessible for a reasonable computational effort. The comparison, on the other hand, revealed that CFD studies nonetheless adequately capture the larger-scale features of the scalar transport, as demonstrated by the close agreement between the dominant eigenmodes of the scalar evolution found numerically and experimentally.

Our study clearly shows that both 3D PTV and 3D LIF are reliable and valuable tools for exploring regimes and features in $3 \mathrm{D}$ realistic mixing flows that are numerically inaccessible. Hence, laboratory setups as those employed here may serve as a prototype for industrial test facilities for detailed experimental exploration and examination of important design parameters of inline mixers, e.g., duct shapes and element geometries in a wide range of flow regimes. In this way, the effects of flow geometry, $R e$ and $P e$ numbers, on the flow and scalar patterns can be studied thoroughly which may allow us to assess the dominance of these design parameters. Moreover, allowing for unsteady effects in the scalar transport and investigating this for different values of $R e$ and $P e$ is an interesting and relevant further topic for future studies.

\section{ACKNOWLEDGMENTS}

This work has been supported by the Dutch Technology Foundation STW (Project No. 11054), which is part of the Netherlands Organisation for Scientific Research (NWO). We thank Paul Bloemen, Jaap M. de Hullu, Henri P. J. Vliegen, Martin J. Huijzer, Freek M. R. van Uittert, Gerald W. J. M. Oerlemans, and Ad P. C. Holten for technical assistance. We also thank Esubalew A. Demissie for sharing the numerical code used for the calculation of the Poincaré map.

\section{APPENDIX: EIGENMODE STRUCTURE OF SCALAR FIELDS}

In 3D spatially periodic flow fields, assuming that the axial flow component is unidirectional, the downstream evolution of the scalar field is dynamically similar to an evolution in time in a $2 \mathrm{D}$ time-periodic flow field. The scalar field $C(x, y, n Z)$, where $Z$ is the non-dimensional period length $(Z=2 L)$ and $n Z$ the integer multiples of $Z$, can then be approximated as a sum of eigenmodes according to

$$
C(x, y, n Z)=\sum_{k=0}^{K} \gamma_{k} \varphi_{k}(x, y) \lambda_{k}^{n}, \quad \lambda_{k}=e^{\mu_{k} Z},
$$

where $\gamma_{k}$ are the expansion coefficients based on initial conditions, $\mathcal{H}_{k}(x, y, n Z)=\varphi_{k}(x, y) \lambda_{k}^{n}$ the Floquet modes, and $\mu_{k}=\sigma_{k}+\mathrm{i} \omega_{k}$ the Floquet exponents with $\mathrm{i}=\sqrt{-1}$. Sets $\left\{\varphi_{k}, \mu_{k}\right\}$ represent the corresponding eigenfunction-eigenvalue pairs governed by the eigenvalue problem

$$
\mathcal{L} \varphi_{k}-\mu_{k} \varphi_{k}=0
$$

where $\mathcal{L}$ is the advection-diffusion operator.

In the case of finite-Pe, the real part of the eigenvalues satisfies $\sigma_{k}<0$, which means that these modes all decay exponentially in time. Ordering the eigenvalues by their real parts following $\ldots<\sigma_{2}<\sigma_{1}<\sigma_{0}=0$ reveals that $k=0$ is a trivial mode and $k=1$ is the slowest non-trivial mode. The latter is the dominant mode that causes the evolution to quickly become governed by the reduced expansion

$$
C(x, y, n Z)=\gamma_{1} \varphi_{1}(x, y) e^{\mu_{1} n Z}+C_{\infty},
$$


where $C_{\infty}=\varphi_{0}$ is the trivial homogeneous eigenmode that corresponds to $\mu_{0}=0$.

In the limit of infinite- $P e$, the transport is equal to the passive advection of a scalar. The fundamental difference with the finite- $P e$ case is that now only purely imaginary eigenvalues (i.e., $\mu_{k}=\mathrm{i} \omega_{k}$ ) occur for all eigenmodes, meaning that none of the eigenmodes will decay. This has the fundamental implication that the evolution becomes of the form

$$
C(x, y, n Z)=\sum_{k} \gamma_{k} \varphi_{k}(x, y) e^{\mathrm{i} \omega_{k} n Z}+C_{\infty},
$$

where the eigenmodes become intimately related to the periodicity and multiplicity of the coherent structures in the Poincare section. Periodic eigenmodes (i.e., $\omega_{k}=2 \pi j / Z$ ) with "low" periodicity (i.e., $j=1,2$ ) dominate the behavior and in that sense act as the dominant eigenmodes. ${ }^{28,29}$ The reason for this dominance of lower-order eigenmodes is that higherorder structures are always embedded in lower-order structures. Lower-order structures therefore correspond to (global) larger-scale features, while higher-order structures correspond to (local) smaller-scale features. ${ }^{29}$

${ }^{1}$ D. G. Baird and D. I. Collias, Polymer Processing: Principles and Design (John Wiley \& Sons, 2014).

${ }^{2}$ M. M. Alvarez, J. M. Zalc, T. Shinbrot, P. E. Arratia, and F. J. Muzzio, "Mechanisms of mixing and creation of structure in laminar stirred tanks," AIChE J. 48, 2135-2148 (2002).

${ }^{3}$ H. A. Stone, A. D. Stroock, and A. Ajdari, "Engineering flows in small devices: Microfluidics toward a lab-on-a-chip," Annu. Rev. Fluid Mech. 36, 381 (2004).

${ }^{4}$ T. Chován and A. Guttman, "Microfabricated devices in biotechnology and biochemical processing," Trends Biotechnol. 20, 116-122 (2002).

${ }^{5}$ J. M. Ottino, "Mixing, chaotic advection, and turbulence," Annu. Rev. Fluid Mech. 22, 207-253 (1990).

${ }^{6}$ V. V. Meleshko, O. S. Galaktionov, G. W. M. Peters, and H. E. H. Meijer, "Three-dimensional mixing in Stokes flow: The partitioned pipe mixer problem revisited," Eur. J. Mech. - B/Fluids 18, 783-792 (1999).

${ }^{7}$ O. S. Galaktionov, P. D. Anderson, G. W. M. Peters, and H. E. H. Meijer, "Morphology development in Kenics static mixers (application of the extended mapping method)," Can. J. Chem. Eng. 80, 604-613 (2002).

${ }^{8}$ R. K. Thakur, C. Vial, K. D. P. Nigam, E. B. Nauman, and G. Djelveh, "Static mixers in the process industries-A review," Chem. Eng. Res. Des. 81, 787-826 (2003).
${ }^{9}$ M. F. M. Speetjens, G. Metcalfe, and M. Rudman, "Topological mixing study of non-Newtonian duct flows," Phys. Fluids 18, 103103 (2006).

${ }^{10}$ V. Kumar, V. Shirke, and K. D. P. Nigam, "Performance of Kenics static mixer over a wide range of Reynolds number," Chem. Eng. J. 139, 284-295 (2008).

${ }^{11}$ M. F. M. Speetjens, E. A. Demissie, G. Metcalfe, and H. J. H. Clercx, "Lagrangian transport characteristics of a class of three-dimensional inlinemixing flows with fluid inertia," Phys. Fluids 26, 113601 (2014).

${ }^{12}$ O. Gorodetskyi, M. F. M. Speetjens, P. D. Anderson, and M. Giona, "Analysis of the advection-diffusion mixing by the mapping method formalism in 3D open-flow devices," AIChE J. 60(1), 387-407 (2014).

${ }^{13}$ H. A. Kusch and J. M. Ottino, "Experiments on mixing in continuous chaotic flows," J. Fluid Mech. 236, 319-348 (1992).

${ }^{14}$ C. Castelain, A. Mokrani, Y. L. Guer, and H. Peerhossaini, "Experimental study of chaotic advection regime in a twisted duct flow," Eur. J. Mech. B/Fluids 20, 205-232 (2001).

${ }^{15}$ G. Metcalfe, M. Rudman, A. Brydon, L. J. W. Graham, and R. Hamilton, "Composing chaos: An experimental and numerical study of an open duct mixing flow," AIChE J. 52, 9-28 (2006).

${ }^{16}$ O. Pust, S. Tyson, P. Mathys, and A. Rütti, "Quantification of laminar mixing performance using laser-induced fluorescence," in 13th International Symposium on Applications of Laser Techniques to Fluid Mechanics, Lisbon, Portugal, 2006.

${ }^{17}$ R. T. M. Jilisen, P. R. Bloemen, and M. F. M. Speetjens, "Three-dimensional flow measurements in a static mixer," AIChE J. 159(5), 1746-1761 (2013).

${ }^{18}$ Wacker Silicone Fluids AK Technical Data Sheet, Amber Composites, 2002.

${ }^{19}$ C. Cuvelier, A. Segal, and A. A. V. Steenhoven, Finite Element Methods and Navier-Stokes Equations (Springer Science \& Business Media, 1986), Vol. 22.

${ }^{20}$ M. Raffel, C. Willert, S. Wereley, and J. Kompenhans, Particle Image Velocimetry: A Practical Guide (Springer, 1998).

${ }^{21}$ J. Willneff, Ph.D. thesis, ETH Zurich, 2003.

${ }^{22}$ B. Lüthi, A. Tsinober, and W. Kinzelbach, "Lagrangian measurement of vorticity dynamics in turbulent flow," J. Fluid Mech. 528, 87-118 (2005).

${ }^{23}$ P. J. Schmid, "Dynamic mode decomposition of numerical and experimental data,” J. Fluid Mech. 656, 5-28 (2010).

${ }^{24}$ P. J. Schmid, "Application of dynamic mode decomposition to experimental data," Exp. Fluids 50, 1123-1130 (2011).

${ }^{25}$ O. Baskan, M. F. M. Speetjens, G. Metcalfe, and H. J. H. Clercx, "Experimental and numerical parametric analysis of a reoriented duct flow," Eur. J. Mech. - B/Fluids 57, 1-14 (2016).

${ }^{26} \mathrm{P}$. V. Danckwerts, "The definition and measurement of some characteristics of mixtures," Appl. Sci. Res. 3, 279-296 (1952).

${ }^{27}$ M. K. Singh, P. D. Anderson, M. F. M. Speetjens, and H. E. H. Meijer, "Optimizing the rotated arc mixer," AIChE J. 54, 2809-2822 (2008).

${ }^{28}$ M. F. M. Speetjens, M. Lauret, H. Nijmeijer, and P. D. Anderson, "Footprints of Lagrangian flow structures in Eulerian concentration distributions in periodic mixing flows," Physica D 250, 20-33 (2013).

${ }^{29}$ M. K. Singh, M. F. M. Speetjens, and P. D. Anderson, "Eigenmode analysis of scalar transport in distributive mixing," Phys. Fluids 21, 093601 (2009). 